No. 1,307.- "On the Stresses of Rigid Arches, Continuous Beams, and Curved Structures."

The Author, in his remarks on M. Gaudard's Paper "On Metal and Timber Arches" read at the Institution of Civil Engineers in December, 1870 , described a method of constructing the curve of equilibrium for an arch unequally loaded, with continuous or discontinuous weights, or under oblique pressure. ${ }^{2}$ The following Paper contains the application of this method to the investigation of the Stresses on Rigid Arches, Continuous Beams, and Curved Structures.

As the consideration of the voussoir arch is more simple than that of the rigid arch, it has been thought desirable to give a preliminary illustration of the method, as applied to the case of an arch of masonry subjected to oblique pressure and to the action of a passing load.

\title{
Voussoir Arch.
}

The arch chosen for examination is that of the Pont-y-tu-Prydd, over the river 'Iafe, in the county of Glamorgan. 'The following particulars are taken from a description of this bridge given in the Minutes of Proceedings of the Institution of Civil Engineers, vol. v., p. 474.

The span is 140 feet, and rise 35 feet. The arch ring is of rubble masonry, of a depth of 2 feet 6 inches on the face, and not more than 1 foot 6 inches in the body of the arch. The first time the bridge was built the arch failed, by the weight of the haunches forcing up the crown. On being rebuilt, the spandrels were lightened by cylindrical openings, and the spaces between these were said to have been filled in with charcoal. It may therefore be taken as a good example of an arch of small stability.

The curves of equilibrium on different suppositions are shown on Plate 3, Fig. 1, the dotted line (b) being drawn as if the spandrels were filled in solid in the usual manner, and the line $(a)$ corresponding to the bridge with cylindrical openings, but without any allowance for the lightness caused by the charcoal filling. It will be observed that the openings aot as negative weights in changing the form of the curve of equilibrium, and bring it to coincide very nearly with the centre of the arch ring; so that the utility of thus lightening the spandrels is unquestionable. This is

1 The discussion upon this Paper occupied portions of two evonings, but an abstract of the whole is given consccutively.

${ }^{2}$ Vide Minutes of Proceedings Iust, C.E., vol. xxxi., pp. 143-148. 
confirmed by an examination of the inverse problem, of finding the load at each point from the form of the curve of equilibrium, assuming it to coincide with the centre of the arch ring.

The dotted black line at the right of Fig. 1 shows the line of roadway on this supposition, and the construction, which may be thus shortly explained, is indicated by the dotted lines.

Having divided the semi-arch into any number of portions, in this case ten, each of the same horizontal length, and drawn vertical lines through the points $p_{1}, p_{2}, p_{3}, \& c$., the centres of these portions, draw the line C D tangential to the curve of the centre line of the arch ring at the crown, to represent the line of horizontal thrust. Since the curve of the arch ring is assumed to be the curve of equilibrium, its tangent at $B$ will cut the line $C D$ in a point $D$ such that $C D=D B$, and this point $D$ will be in the vertical line passing through the centre of gravity of the unknown mass of the arch between $\mathrm{B}$ and $\mathrm{C}$.

Draw any horizontal line $\mathrm{D}^{\prime} \mathrm{E}$, and lines $\mathrm{D}^{\prime} q_{\mathrm{t}}, \mathrm{D}^{\prime} q_{2}, \mathrm{D}^{\prime} q_{\mathrm{s}}, \& \mathrm{c}$, through $\mathrm{D}^{\prime}$ respectively parallel to $p_{1} p_{2}, p_{2} p_{3}, p_{3} p_{4}$, \&c.; also draw the line $\mathbf{E} \mathbf{F}$ vertical, at such a distance $\mathbf{E} \mathrm{D}^{\prime}$ from $\mathrm{D}^{\prime}$ that $\mathrm{E} q_{1}$, the part of it cut off by the line $\mathrm{D}^{\prime} q_{1}$, may be equal to the height from the soffit to the roadway at the point $p_{1}$, and will thus measure the weight of that portion of the arch acting at $p_{1}$. Then $q_{1} q_{2}, q_{2} q_{3}$, $\& c$, the parts of the vertical line through $E$, cut off by the lines $D^{\prime} q_{1}, D^{\prime} q_{2}$, \&c., will be the heights of the roadway above the soffit at the points $p_{2}, p_{3}, \&$ c., and if these be set up vertically above the soffit at the different points, the curve line connecting their upper extremities will be the required line of roadway, which, it will be observed, falls considerably below the line of the actual roadway at the part near the openings.

In drawing the above lines, the arch has been divided into portions having equal horizontal lengths, because then the weight of each portion is measured by the height from the soffit to the roadway. This supposes the density of the backing to be the same as that of the voussoirs, which is probably not far from the truth. The pressure has been considered as wholly vertical, or the backing as composed of a number of separate vertical prisms with horizontal bases, through which the pressure is transmitted to the voussoirs, the backs of which may be considered as notghed in level steps, to receive the bases of the prisms.

But the material of the backing must have some amount, however small, of mobility among its particles, and the back of the arch having an inclined surface, the pressure cannot be quite vertical. In the absence of any information as to the nature of the 
backing, the small deviation of the pressure from verticality cannot be known, but some idea of its effect may be gained by ascertaining the nature of the change of the curve of equilibrium, on the supposition that the backing, while retaining its density, is a perfect fluid, pressing at right angles to the back of the arch.

Having divided the arch ring into portions of equal horizontal lengths, as a sufficient approximation, the whole mass of each portion may be taken as pressing at right angles to the back of the arch, with a head equal to the height between the soffit and the roadway at the centre of the division.

At any point $p$, on the left side of Fig. 1, the intersection of the vertical line through the centre of one of the divisions with the centre line of the arch ring, take $p s=p^{\prime} s^{\prime}$, and draw the normal line $p t$, and horizontal line $s t$. Then $p s$ will represent the weight of the portion acting at $p, s t$ its horizontal push, considered as a fluid with a head equal to $p s$, and $p t$, normal to the curve, will be the resultant of these two forces.

The horizontal forces acting at the different points are thus found, and from these, by the method of moments, the vertical height above $A$ of the line of action of their resultant or sum can be easily ascertained. Draw $M N$ at this height, and from the point $\mathbf{M}$; where the vertical line $G \mathbf{M}$ through the centre of gravity of the semi-arch cuts $M N$, set off $M N$ equal to the sum of the horizontal forces, and draw N O vertical and equal to the weight of the half arch. The line $\mathrm{M} \mathrm{O}$ will then be the resultant of all the forces acting on the semi-arch, and will be in the direction of the normal to the curve of the arch ring, since the direction of each of the component forces is normal. The line $\mathrm{M} \mathrm{O}$ will cut the horizontal line through $\mathrm{C}$, the centre of the crown of the arch in some point $G^{\prime}$, and the points $G^{\prime}$ and $A$, being joined, $G^{\prime} A$ will be the direction of the thrust at $A$. To find its amount, draw any vertical line $r k$ equal to the weight of the half arch, and draw a horizontal line $\mathrm{K} k \mathrm{~L}$ through the point $k$. From any point $\mathrm{G}$ in $\mathrm{C} \mathrm{G}^{\prime}$ draw $\mathrm{G} \mathrm{L}$ parallel to $\mathrm{G}^{\prime} \mathrm{A}$, cutting $\mathrm{K} k$ in $\mathrm{L}$, and from $L$ draw $L H$ parallel to $G^{\prime} O$, cutting $C$ G produced in $H$. Then $\mathrm{H} \mathrm{L}$ will be equal to the resultant $\mathrm{M} O$, and $\mathrm{G} L$ will be the amount of the thrust at $\mathrm{A}$.

To draw the curve of equilibrium, set off from $\mathrm{H}, \mathrm{H} v_{1}$ equal and parallel to the force acting at, $p_{1}^{\prime}, v_{1} v_{2}$ equal and parallel to the force at $p_{2}^{\prime}$, and continue this construction, which, if correct, will terminate by the end of the last line drawn coinciding with the point $\mathbf{L}$. This force diagram may also be made by 
plotting the vertical and horizontal components of the separate forces at $p_{1}{ }^{\prime}, p_{2}{ }^{\prime}$, \&c., beginning at the point $H$. The curve of equilibrium can then be constructed by drawing from the point $p_{1}{ }^{\prime}$, where the direction of the force at $p_{1}^{\prime}$ cuts the horizontal line $\mathrm{CH}$, $p_{1}^{\prime} z_{2}$ parallel to $\mathrm{G} v_{1}$, to cut the direction of the force acting at $p_{2}{ }^{\prime}$ in the point $z_{2}$; from $z_{2}$ thus found drawing the line $z_{2} z_{3}$ parallel to $G^{\prime} v_{2}$, to cut the direction of the force at $p_{3}{ }^{\prime}$ in $z_{s}$, and continuing this construction, which will terminate by the last line drawn passing through the point $A$, and coinciding with $A G^{\prime}$.

The supposition of fluid backing shows a considerable alteration of the curve of equilibrium, and indicates the tendency of the obliquity of the pressure of ordinary backing to make this curve deviate towards the extrados at the haunches of the arch. In the present instance, the effect of the lightness of the charcoal filling . at the openings would be to cause a deviation in the opposite direction; so that, in all probability, the true curve is represented by the line (a), and the arch has almost all the stability which an arch ring of such small depth is capable of.

In order to obtain a somewhat more definite idea of the stability, it is not difficult to ascertain what change will take place in the curve of equilibrium when a load of known weight passes over the bridge.

It will be explained hereafter that the effect of a load is greatest when it acts about midway between the crown and the abutment, and that the curve of equilibrium for a load considered as the only force acting on the arch is represented by two straight lines, one of which passes through the crown of the arch and the centre of the abutment farthest from the load, and the other line passes through the abutment nearest to the load, and the point where the vertical line through the load outs the first-mentioned line.

Further, by Equation (13), page 89, the curve of equilibrium of the unloaded arch will by the loading be altered at every point through a space equal to the vertical distance between the neutral line of the arch and the line of equilibrium of the load, this distance being reduced in the ratio of the horizontal thrust of the load to that of the arch and load.

Draw, therefore, the vertical line $c d$ through the load supposed to act at $e$, cutting $\mathrm{B} \mathrm{C}$ produced in $d$, and join $d \mathrm{~A}: d \mathrm{~A}, d \mathrm{~B}$ will be the curve or lines of equilibrium of the load alone, and if $a b$ be drawn vertically, and equal to the load, and $d g$ horizontally to cut $a b$ in $g, d g$ will be the horizontal thrust caused by the weight alone. To reduce this to numerical quantities, if in the present 
instance a load of 5 tons be supposed to act in the line $c d$, the horizontal thrust will be $2 \cdot 4$ tons.

'The weight of the semi-arch may be taken at 415 tons, and its horizontal thrust estimated by scale from Fig. 1 at 309 tons.

If the vertical ordinates botween the curve of the arch and lines of equilibrium of the weight be reduced in the above-mentioned ratio, and superposed on the curve of equilibrium of the arch itself, the alteration of the curve at the point under the weight will, when reduced in the above-mentioned ratio, be $28 \cdot 5$ feet $\times \frac{2 \cdot 4}{8114}$, or say 3 inches. In like manner, the depression in the middle of the opposite semi-arch will be 10 feet $\times \frac{2 \cdot 4}{5114}$, or say 1 inch.

When a weight of 5 tons, therefore, acts on the arch midway between the crown and the abutment, the curve of equilibrium will be raised 3 inches at the part where the weight acts, and depressed 1 inch at the middle of the opposite semi-arch.

As the former deviation is nearly $\frac{1}{6}$ th of the thickness of the arch ring, the compression per square inch will, by the remarks on Equation ( $6 a$ ) page 74 , as compared with the uniform comprossion if the curve of equilibrium were central, be doubled at the extrados, and reduced to zero at the intrados of the arch.

Taking the depth of the arch ring at 20 inches, and its width at the crown at 14 feet 6 inches, the uniform compression there caused by the horizontal thrust of 309 tons is nearly 13 tons, and at the point where the weight is supposed to act 14 tons per square foot.

During the passage of a load of 5 tons over this bridge, therefore, the extrados at the point under the weight would be sustaining a compression of 28 tons per square foot. This result would be to some extent mitigated by the pressure from the weight being conveyed to the arch through the backing, and thus acting on a surface of some extent, instead of a point as supposed.

Friction would not modify the curve of equilibrium, but it would be of service in preserving the stability of the arch, if in any part the workmanship were so faulty that the joints of the arch were inclined to the normal at any angle less than the angle of friction.

The cohesion of the mortar, after it had properly set, like the elastic forces of a rigid arch, would be brought into action if the curve of equilibrium deviated from the centre line of the arch ring by more than $\frac{1}{6}$ th of its depth. There would then be a tensile stress on the opposite edge of the arch ring, and the cohesion of 
the mortar would resist this, "and diminish to some extent the compression at the other edge.

The nature of the alteration of the curve of equilibrium, on the supposition of the fluidity of the backing, shows that the practice sometimes adopted, of coating the back of an arch with puddle, may be useful in segmental arches of large rise and level roadway, besides making them watertight. In these, the curve of equilibrium near the abutments descends below the curve of the arch ring, and the partial fluidity of the backing would tend to raise the curve and increase its curvature at these points. There would also be less horizontal thrust against the abutments, since the thrust at $A$ is altered from $A G$ to $A G^{\prime}$ by the supposition of the fluidity of the backing. There would thus be a tendency to diminish any injurious stress on the arch and abutments until the mortar had properly set.

\section{RigID ARCH.}

The investigation of the stresses of a rigid arch has hitherto been a subject of considerable difficulty, owing to the intricate nature of the mathematical analysis it is necessary to employ.

The circular rib has been examined by Mr. Wilfrid Airy, Assoc. Inst. C.E., in his recent treatise on "Iron Arches," and M. Gandard has given, in the Appendix to his Paper on "Metal and Timber Arches" (Min. of Proc. Inst. C.E., vol. xxxi., p. 98), a solution of the case of a circular rib of varying section. The subject has also been investigated generally, and applied in some detail to the case of the parabolic rib, by Dr. Rankine, in his work on Civil Engineering. The results of these authors being expressed in algebraical formula, the labour of applying them numerically to trace the variation of stress from point to point is considerable. Still, before the transverse sections of arch ribs can be proportioned to the stresses coming upon them, a knowledge of this variation is indispensable.

In experiments on the strength of materials, it is found that there are considerable differences in the breaking weights of specimens of the same kind of material, although the dimensions of the specimens are identical. This fact, which makes it necessary to employ a large factor of safety in engineering constructions, renders it unnecessary to solve questions of stress with minute accuracy, and the graphic method of solution is generally a sufficient approximation in practice. From the assistance which this method affords in the treatment of voussoir arches by means 
of the curve of equilibrium, it occurred to the Author that if it could be applied to the investigation of rigid arches, an equal facility of solution would be obtained.

It will now be shown that the method can be applied, and that the stresses at every point of an arched rib admit of being represented by a diagram, so that their changes from point to point can be readily ascertained. Some questions, also,.such as those where the form of the rib differs from the circle and parabola, and where the pressure is oblique, which would be almost intractable by analysis, can be readily solved by this method.

The curve of equilibrium of an arch is so constructed, that the pressure at any point in the direction of the curve is combined with the external force acting there. The resultant pressure is in the altered direction of the curve beyond the point. By this construction it will be seen, that the curve is the locus of the resultant of all the outward forces, and since the moment of a resultant is equal to the sum of the moments of its component forces, the 'bending moment,' or transverse stress at any point, is equal to the pressure in the direction of the tangent to the curve of equilibrium, multiplied by the perpendicular on this tangent, from the point in question. This moment tends to bend the rib in different directions, according as the curve of equilibrium is above or below the neutral line of the rib, or line passing through the centres of gravity of its transverse sections.

A general idea of the nature of the stresses may be given by Figs. A and B, page 65, premising, as will be shown hereafter, that a force acting parallel to the neutral line, at a given perpendicular distance from it, may be considered as equivalent to a force of the same amount acting in the neutral line, together with a couple, whose moment is the force multiplied by the given perpendicular distance from the neutral line.

The absolute amounts of the stresses may be ascertained by Equation ( $6 a$ ), page 74 .

1st. The rib is subject to a general compression throughout its whole length, to the same extent as if it were a voussoir arch, and the curve of equilibrium coincided with its neutral line. This may be represented by a force $\mathbf{F}$ (Fig. A) uniformly distributed over the area of section, and giving rise to the equal forces per square inch represented by the lines $f, f, f$ acting at the different points of this area.

2nd. The rib is subject also to the stress caused by the bending moment. When this acts so as to increase the curvature, in which case the curve of equilibrium descends below the neutral 
line of the rib, its state of stress may be represented as in Fig. A.

Any transverse section A B of the rib A B C D is in the same Fig. A.

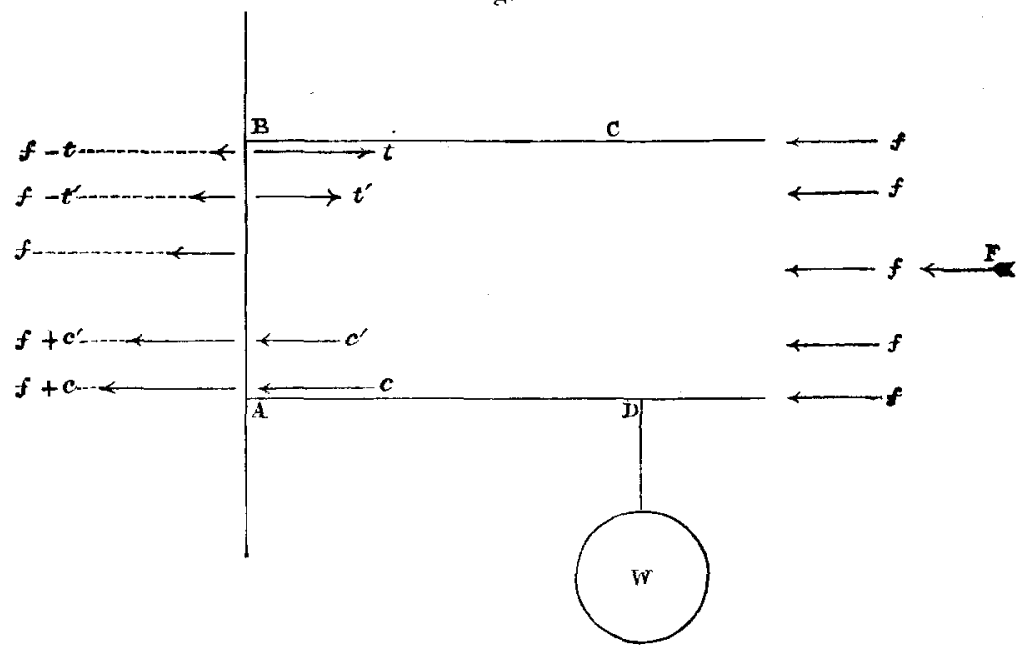

Fig. B.

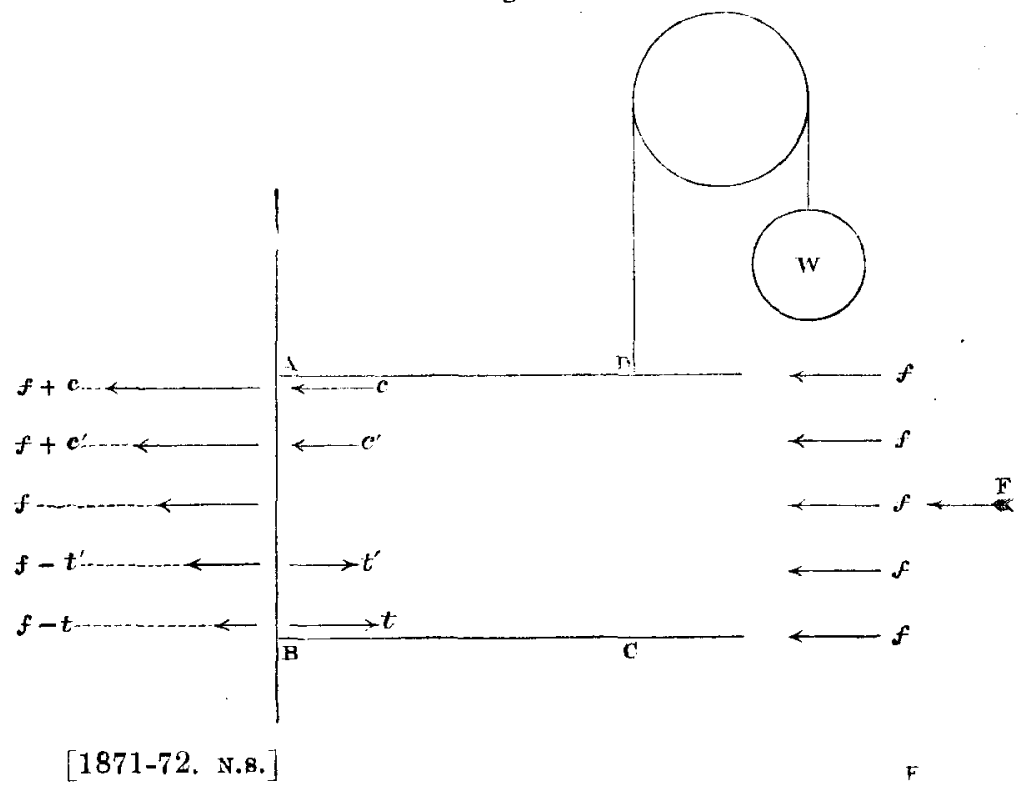

Downloaded by [ University of Sussex] on [15/09/16]. Copyright @ ICE Publishing, all rights reserved. 
condition as if this section were fixed in a wall, and acted on by the force $F$, and also by a weight $W$ at a leverage $A D, W \times A D$ being equal to the bending moment, and the total stress at any point is the algebraical sum of the stress due to the action of $\mathrm{F}$, and of that due to the bending moment. This latter will excite tensile forces at the top, and compressive forces at the bottom of the rib, which forces per square inch may be represented on the diagram, Fig. A, by the lines $t, t^{\prime}, c^{\prime}, c$. The result of the actions of $\mathbf{F}$ and $\mathrm{W}$ together is that the stresses at the top are represented by the lines $f-t, f-t^{\prime}, \& \mathrm{c}$, and at the bottom by $f+c, f+c^{\prime}, \& \mathrm{c}$.

In like manner, at those points of the rib where the bending moment tends to diminish the curvature, and the curve of equilibrium is above the neutral line of the arch rib, the transverse section $A B$ is in the state represented by Fig. B, the weight $W$ acting upwards instead of downwards, as in Fig. $A$.

Here the tensile forces are at the bottom, and the compressive forces at the top of the rib, the forces resulting from the action of both $\mathrm{F}$ and $\mathrm{W}$ being $f+c$ at the top, and $f-t$ at the bottom.

The values of $c$ and $t$ being independent of $f, f-t$ may become either zero or negative; that is, the part of the rib to which $f-t$ corresponds may either have no strain or be in tension, according as the tensile force excited at the outer surface by the bending moment is equal to, or greater than, the uniform compressive force $f$ caused by the action of $\mathrm{F}$.

In the state represented by Fig. A, the bending moment will in what follows be considered as positive, or tending to increase the curvature of the rib, and in the state represented by Fig. $B$ as negative, or tending to diminish the curvature.

\section{General Conditions of Equilibrium.}

For the voussoir arch, the transverse stress which could be overcome by the cohesion of the mortar being so small as not to be worth considering, the curve of equilibrium has generally been assumed to pass through the centre, or, at all events, within the substance of the arch ring at the crown, and its position there determines the horizontal thrust.

But the mode of constructing the curve of equilibrium is independent of its passing through any particular points, either at the crown or at the springing. The points there may be shifted either upwards or downwards, and the curve can be constructed in exactly the same manner, but its figure will be altered by varying the position of these points. 
It is necessary to explain the meaning of these changes of position.

When the vertex of the curve at the centre of the crown is raised above the neutral line, it appears by the previous remarks as to the moment of the resultant, that a bending moment will exist there, tending to diminish the curvature of the rib. The horizontal thrust will also be diminished, since it acts at a greater vertical distance from the abutment.

Supposing the vertex of the curve of equilibrium to recede upwards from the crown of the arch, the horizontal thrust will continue to diminish, but its leverage to increase. The bending moment at the crown of the arch may therefore be finite, even when the vertex recedes to an infinite distance. The condition, in fact, then corresponds to that of a girder resting on level abutments, and incapable of supplying any horizontal thrust.

On the other hand, if the vertex of the curve descends below the crown of the arch, the bending moment there becomes positive, and tends to increase the curvature of the arch rib. The horizontal thrust is also increased, the weight of the arch remaining the same. When the horizontal thrust is finite, but the weight and rise of the arch are both equal to zero, then the arch becomes a horizontal pillar.

When the springing points' of the curve are shifted, so that the eurve does not pass through the centre of the arch ring at the abutments, a bending moment, of an amount determined by the alteration of the springing points, exists at the abutments, and the horizontal thrust is also altered.

By thus shifting the position of the vertex, or of the springing points, or both, of the curve of equilibrium, the horizontal thrust, and the transverse stress at the crown and springing may be indefinitely varied, and may be made to satisfy the conditions of a rigid arch with the feet kept from spreading, so that the span is invariable, or of a rigid arch with the ends 'fixed,' or "encastré."

What these conditions are will now be explained; but it is necessary to offer some elementary remarks as to the effect of transverse stress on the curvature of an arch rib.

In Fig. $C$, if $A B$ be a small portion of the neutral line of an arch rib, its centre of curvature being at $O$, when it is under transverse strain, the line A B will change to some other position, as $\mathbf{A ~ B}^{\prime}$, the centre of curvature changing to $O^{\prime}$. And a concentric length $\mathrm{CD}$, distant from the neutral line by the length $\mathrm{CA}$, 
will become curved into $\mathrm{CD}^{\prime}$, and will be elongated or contracted according as the point $\mathrm{C}$ is farther from or nearer to the centre of curvature than the point $A$.

Fig. $C$.

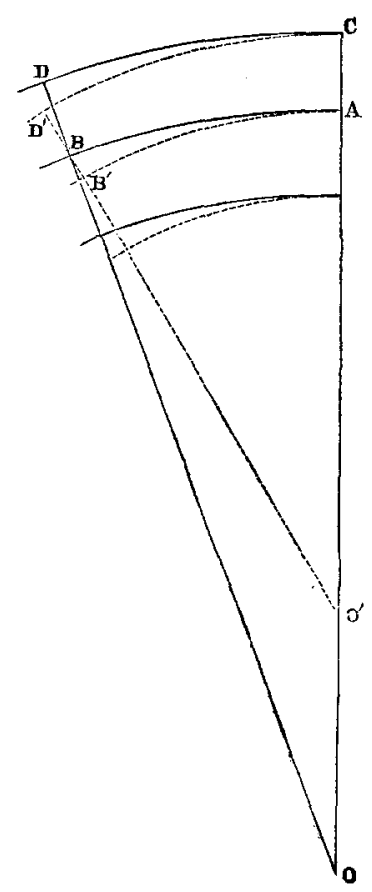

The ratio of this change of length $\mathrm{CD}^{\prime}-\mathrm{CD}$ to the primary length $\mathrm{CD}$ measures the force exerted by the fibre CD. If $a$ be the reciprocal of the fraction which expresses the extension of the unit of length of the material by a force of 1 ton per square inch, then $\alpha \cdot \frac{\mathrm{CD}^{\prime}-\mathrm{CD}}{\mathrm{CD}}$ will be the force exerted by $\mathrm{C} \mathrm{D}$ considered as having a sectional area of unity. And if the transverse section of the rib be divided into a number of small portions, each having an area $=\Delta A$, $A$ being the total area, and the area of $C D$ be one of these portions, then $a \cdot \frac{\mathrm{CD}^{\prime}-\mathrm{CD}}{\mathrm{CD}} \cdot \Delta \mathrm{A}$ will be the force exerted by C D, considered as having the area $\Delta A$.

But $\mathrm{CD}-\mathrm{AB}: \mathrm{AB}:: \mathrm{CA}: \mathrm{AO}$

or $C D-A B=A B \frac{C A}{A O}$ and $\mathrm{CD}^{\prime}-\mathrm{AB}^{\prime}: \mathrm{AB}^{\prime}:: \mathrm{CA}: \mathrm{AO}^{\prime}$ or $C D^{\prime}-A B^{\prime}=A B^{\prime} \frac{C A}{A O^{\prime \prime}}$.

and deducting, since $\mathrm{AB}=\mathrm{AB}$,

$$
C D^{\prime}-C D=A B\left(\frac{C A}{A O^{\prime}}-\frac{C A}{A O}\right)
$$

Or, taking the limit of $\frac{\mathrm{AB}}{\mathrm{CD}}=1$,

$$
\frac{\mathrm{CD}-\mathrm{CD}}{\mathrm{CD}}=\mathrm{CA}\left(\frac{1}{\mathrm{AO}^{\prime}}-\frac{1}{\mathrm{AO}}\right)
$$

The force exerted by $\mathrm{CD}$ is, therefore, in tons,

$$
\alpha\left(\frac{1}{\mathrm{AO}^{\prime}}-\frac{1}{\mathrm{AO}}\right) \mathrm{CA} \cdot \Delta \mathrm{A},
$$

and is thus proportional to the difference between the reciprocals of the radii of curvature $\mathrm{AO}, \mathrm{AO}^{\prime}$. 
The moment of this force with respect to the neutral axis is found by multiplying it by $\mathrm{CA}$, or is $a\left(\frac{1}{\mathrm{AO}^{\prime}}-\frac{1}{\mathrm{AO}}\right) \mathrm{CA}^{2} \cdot \Delta \mathrm{A}$.

Calling $M$ the moment of the whole sectional area,

$$
\mathrm{M}=a\left(\frac{1}{\mathrm{AO}^{\prime}}-\frac{1}{\mathrm{AO}}\right) \cdot \Sigma \mathrm{CA}^{2} \cdot \Delta \mathrm{A} .
$$

The last factor $\mathrm{SC} \mathrm{A}^{2} \cdot \Delta \mathrm{A}$, or the sum of all the small portions of the area, each multiplied by the square of its distance from the neutral axis, is generally represented by $\mathbf{I}$.

Hence,

$$
\mathrm{M}=a \mathrm{I}\left(\frac{1}{\mathrm{AO}^{\prime}}-\frac{1}{\mathrm{AO}}\right) . . . .
$$

But calling $t_{0}$ the tension or compression per square inch at the outer surface of the rib, considering CD as this outer surface, and putting $\mathrm{C} \mathrm{A}=k_{0}$

$$
\frac{\mathrm{CD}^{\prime}-\mathrm{CD}}{\mathrm{CD}}=\frac{t_{0}}{\alpha}=k_{0}\left(\frac{1}{\mathrm{AO} O^{\prime}}-\frac{1}{\mathrm{AO}}\right)
$$

and, by combining this with (1),

$$
\mathbf{M}=\frac{t_{0}}{k_{0}} \cdot \mathbf{I} \cdot \text {. . . . . . . . }
$$

This moment M, representing the moment of the elastic forces of the rib, is at every point in equilibrium with the moment of the outward forces.

Fig. D.

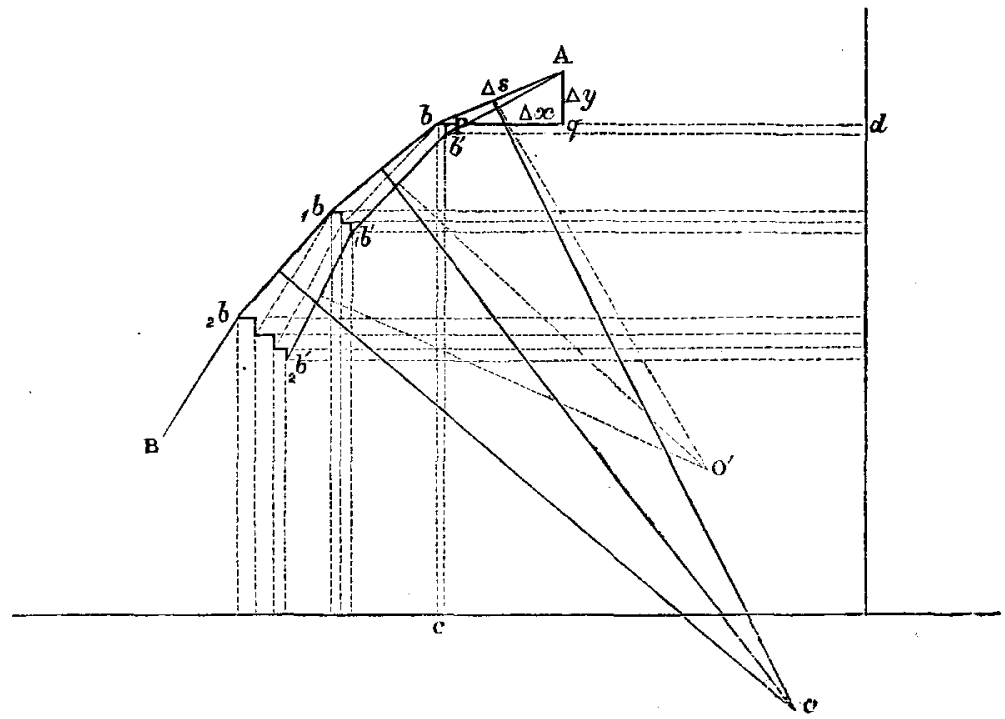


In Fig. D, let $\mathrm{A} b=b_{1} b={ }_{1} b_{2} b$, \&c., each of these being equal to $\Delta s$, be a polygon forming the chord lines of a portion of the neutral curve of an arch rib before strain, the centre of curvature being $O$.

When the rib is strained, the polygon will change into some other form as $\mathrm{A} b^{\prime}{ }_{1} b^{\prime}, \&$ c., the centre of curvature changing to $O^{\prime}$.

If $\Delta s=\mathrm{A} b$ subtend an angle $\theta$ at the centre $O$ before strain, and an angle $\theta^{\prime}$ at the centre $O^{\prime}$ after strain, then

$$
\theta=\frac{\Delta s}{\mathrm{~A} \bar{O}} \quad \theta^{\prime}=\frac{\Delta s}{\mathrm{~A} \bar{O}^{\prime}}
$$

and

$$
\begin{aligned}
\theta^{\prime}-\theta & =\Delta s\left(\frac{1}{\mathrm{AO}^{\prime}}-\frac{1}{\mathrm{AO}}\right) \\
& =\Delta s \frac{\mathrm{M}}{a \mathrm{I}}
\end{aligned}
$$

by equation (1).

Since lines drawn through the middle parts of these chords, and perpendicular to them, meet in the centre of curvature, the angle $\theta^{\prime}-\theta$ is equal to the angle $b^{\prime} \mathrm{A} b$, and expresses the change of direction of $\Delta s$ after the rib has been strained.

The total change of direction of the tangent to the curve of the rib between the points $A$ and $B$ will therefore be the sum of all the values of $\theta^{\prime}-\theta$ between these limits. Calling this change of angle $\Theta$,

$$
\Theta=\Sigma_{\Delta}^{B} \frac{1}{a I} M \Delta s .
$$

And if, as will be supposed in all the subsequent examples except the last, the section of the rib be uniform throughout the length, the value of I will be constant, and it may be set outside the sign of summation, or

$$
\Theta=\frac{1}{a I} \Sigma_{\Lambda}^{\mathrm{B}} M \Delta s .
$$

The neutral line of the arch rib having been divided into equal lengths, each equal to $\Delta s$, and the value of $M$ being known for each of these portions, $\Theta$ is found by summing all these values of $\mathbf{M}$ between the limits $\mathbf{A}$ and $\mathbf{B}$, and multiplying this sum by

$$
\frac{\Delta s}{a \mathrm{I}}
$$

If the arch rib be 'fixed' at the points $A$ and $B$, the curve of the rib is not at liberty to change its direction there by the action 
of the outward forces, and therefore in this case $\Theta=0$; or, if the rib be fixed at the ends, the condition

$$
\Sigma_{\Delta}^{B}(M)=0 \text {. }
$$

must be fulfilled, and the curve of equilibrium must be so chosen as to satisfy this condition.

Further, the length of the line $b b^{\prime}$, which measures the change in the position of the end of $\Delta s$, is $\Delta s\left(\theta^{\prime}-\theta\right)$,

or

$$
b b^{\prime}=\Delta s^{2} \frac{\mathrm{M}}{a \mathrm{I}} \text {. }
$$

Referring the curve to rectangular axes, and calling $b q=$ $\Delta x, \mathrm{~A} q=\Delta y$, the change $b b^{\prime}$ may be considered as made up of the horizontal change $b p$, and the vertical change $b^{\prime} p$.

By similar triangles, $b b^{\prime}$ being small,

$$
\begin{aligned}
b p & =b b^{\prime} \frac{\Delta y}{\Delta s} \\
& =\frac{1}{\alpha} \mathrm{I}^{-} \mathrm{M} \Delta s \Delta y \\
b^{\prime} p & =b b^{\prime} \frac{\Delta x}{\Delta s} \\
& =\frac{1}{a \mathrm{I}} \cdot \mathrm{M} \cdot \Delta s \Delta x .
\end{aligned}
$$

Therefure if $h$ and $v$ be the horizontal and vertical displacements of the point $B, I$ being supposed constant,

$$
\begin{aligned}
& h=\frac{1}{a \mathrm{I}} \Sigma \Sigma \mathbf{M} \Delta s \cdot \Delta y \quad . \quad . \quad . \quad . \quad \text {. } \\
& v=\frac{1}{\alpha \mathrm{I}} \Sigma \mathbf{\Sigma} \mathbf{M} \Delta s \cdot \Delta x \quad . \quad . \quad . .
\end{aligned}
$$

the summations being made between the limits $\mathrm{A}$ and $\mathrm{B}$.

Fig. $D$ is intended to show the building up of the displacement at $\mathrm{B}$, by the displacements at $b,{ }_{1} b,{ }_{2} b$, \&c.

Referring to Equation (4), the double summation may be made in two ways:

(1). By forming for each point $b$ the sum of all the values of $\mathbf{M}$ from the limit A up to this point, multiplying this sum by the value of $\Delta y$ at the point, and summing up these produots for all the points between $\mathbf{A}$ and $\mathbf{B}$.

(2). Since the sum $\Sigma \Delta y=y=b c$ for each point, the summation may be made by forming the sum $\mathbf{\Sigma}(\mathbf{M} y)$ or $\mathbf{\Sigma}(\mathbf{M} \cdot b c)$ between the limits $\mathrm{A}$ and $\mathrm{B}$. 
The latter, which is that used in Mr. W. Airy's treatise, is tho casier one, and is better adapted to the arrangement, here followed, of considering the forces on each element of arc, and the bending moment $M$, to act at the centre of the elementary arc.

It consists, therefore, of the following process :

Having found the values of $\mathbf{M}$ for each part $\Delta s$, multiply each value of $M$ by the ordinate $b c$ at the point to which it corresponds, and add all these products together between the limits $A$ and $B$.

If the feet of the arch rib be kept from spreading, the horizontal displacement is zero, and the curve of equilibrium must satisfy the condition

$$
\mathbf{\Sigma}(\mathbf{M} \cdot b c)=0 \quad \text {. . . . . }
$$

between the limits of the ends of the arch rib.

And if the arch rib is also fixed at the abutments, so that not only the span is invariable, but the angle contained between the tangents to the curve at each abutment is also invariable, the conditions (3) and (6) must coexist,

or

$$
\begin{aligned}
\mathbf{\Sigma}(\mathbf{M}) & =0 \\
\mathbf{\Sigma}(\mathbf{M} \cdot \boldsymbol{b} \boldsymbol{c}) & =0 .
\end{aligned}
$$

In this case, the curve of equilibrium generally does not pass either through the crown or the ends of the arch rib, but the vertex and springing points of the curve will require to be altered until the two conditions are satisfied.

These conditions also co-exist if the rib be fixed in direction at one end, and the other end be fixed in position, though not in direction, so that the span remains invariable. The curve of equilibrium will pass through that end of the rib which is only fixed in position, the bending moment being there equal to zero, and the vertex, and springing point of the curve at the other end of the rib, will require to be raised or lowered until the above conditions are satisfied.

Another arrangement, which is virtually a pair of cantilevers meeting in the middle of the span, is where the rib is fixed at the abutments and hinged at the crown. In that case, the curve of equilibrium must pass through the crown, and the springing points be raised so as to satisfy the condition

$$
\Sigma(\mathbf{M} \cdot b c)=0 .
$$

Fitherto the section of the rib has been considered as uniform thronghout the whole length. If it is desired to consider the section as changing from point to point, the quantity I which has 
heen taken as constant must be replaced under the sign of summation, or the values of $\frac{\mathrm{M}}{\mathrm{I}}$ must be used instead of $\mathrm{M}$ in satisfying the conditions.

Conditions (3) and (6) will then be replaced by

$$
\begin{aligned}
& \mathbf{\Sigma}\left(\frac{\mathrm{M}}{\mathrm{I}}\right)=0 \\
& \mathbf{\Sigma}\left(\frac{\mathrm{M}}{\mathrm{I}} \cdot b c\right)=0 .
\end{aligned}
$$

And the value of $M$ for each length $\Delta s$ must be divided by $I$ before entering it in the sum which is to be made equal to zero between the limits.

To construct a rib with sections at each point proportioned to the stresses, a uniform sectional area may be assumed as a first approximation. The curve of equilibrium being then drawn and the stresses determined, the rib may next be assumed to have sections proportioned to these stresses as a second approximation.

The curve of equilibrium being again drawn, will differ slightly from the previous one, inasmuch as $\frac{M}{\mathrm{I}}$ has been used in fixing it instead of M. The stresses being then determined, another approximation to the correct sections will be obtained by making them proportioned to the altered stresses, and will probably be near enough for practical purposes, but the approximation may be carried as far as thought necessary.

Having determined the conditions which the curve of equilibrium must satisfy, it is not difficult to construct the curve after a few trials by the method mentioned at the beginning of this Paper. The value of the bending moment or transverse stress at any point thus becomes known.

\section{Effect of Bending Moment, and Amount of Total Stress.}

In order to appreciate the effect of the addition of a bending moment on the stress of an arched rib, let $F$ be the resultant pressure in the direction of the tangent to the curve of equilibrium, which may, for the purpose of this illustration, be supposed parallel to the tangent to the neutral line of the rib, or the uniform compressive force if the rib be in the state of the voussoir arch (Figs. A and B); and let $p$ be the perpendicular let fall on the direction of $\mathrm{F}$ at the point of the rib to which $F$ corresponds. The addition of two forces $+\mathrm{F}^{\prime}$ and $-\mathrm{F}^{\prime}$, each 
equal in amount to $\mathbf{F}$, but acting in opposite directions, at that point of the neutral line of the rib to which $\mathrm{F}$ corresponds, the directions being coincident with the tangent to the neutral line, will not disturb the equilibrium; and $\mathrm{F}$ may be considered as equivalent to $+F,+F^{\prime \prime}$ and $-F^{\prime \prime}$. The effect of $+F$ and $-F^{\prime \prime}$ is to cause a bending moment, and, since $F^{\prime}=F$, the effect of the remaining force $+F^{\prime}$ is to cause a direct compression of the rib, of the same amount as if $\mathbf{F}$ acted in the neutral line. The bending moment

$$
\mathbf{M}=\mathbf{F} p \text {; }
$$

and if $\mathrm{I}$ is put equal to $\mathrm{A} q^{2}$, where $\mathrm{A}$ is the area of cross section and $q$ its radius of gyration in reference to the neutral axis, then by formula (2),

and

$$
\begin{aligned}
\mathrm{F} p & =\frac{t_{0}}{k_{0}} \mathrm{~A} q^{2}, \\
t_{0} & =\frac{\mathrm{F}}{\mathrm{A}} \cdot \frac{k_{0} p}{q^{2}}
\end{aligned}
$$

for the tension or compression per square inch arising from the action of the bending moment, or what has been called the forces $t$ and $c$ in Figs. A and $B$.

The total stress being that due to the direct action of $\mathrm{F}$ and a couple represented by $\mathbf{F} p$, the rib will sustain a direct compression from $F$ as well as the force $t_{0}$. This compression, considered as uniformly distributed over the area $A$ of section, will give a compression per square inch of $\frac{F}{A}$, and the total stress will be

$$
\frac{\mathrm{F}}{\mathrm{A}}+\frac{\mathrm{F}}{\mathrm{A}} \cdot \frac{k_{0} p}{q^{2}}
$$

or, since $\frac{F}{A}$ is what has been called the force $f$ in Figs. $A$ and B,

$$
\text { Total stress }=f\left(1+\frac{k_{0} p}{q^{2}}\right) \cdot . \quad . \quad . \quad . \quad . \quad \cdot(6 a)^{1}
$$

the positive sign representing compression, and the negative sign tension.

If $p$ be taken $=k_{0}$, that is, if a point in the arch rib be considered for which the curve of equilibrium is approximately

' This formula may be deduced from the usual considerations in regard to elasticity, in such a manner as to lead to some interesting conclusions.

Let $A B C D$ in the annexed Fig. be a portion of the rib or solid acted on by the force $F$, the plane $A B C D$ passing through $G$ the centre of gravity of the 
coincident with the outer surface, the above value of the stress becomes

$$
f\left\{1+\left(\frac{k_{0}}{q}\right)^{2}\right\}
$$

area of cross section, and $\mathbf{Q} \mathbf{F}$ the direction of the force $\mathbf{F}$. Also, let $\mathbf{A} \mathbf{B}$ perpendicular to $Q \mathrm{~F}$ be the intersection of $\mathrm{ABC}$, and the plane of cross section.

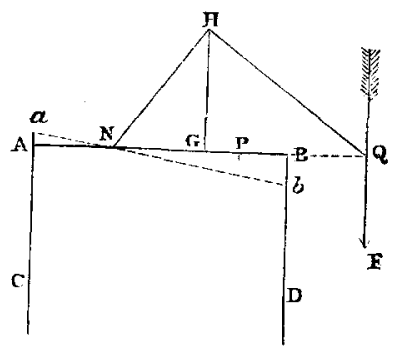

The area of cross section of the solid is supposed to be perpendicular to the plane of the paper, and of any form whatever. Let $Q$ be the point where the line of direction of the force $F$ cuts $A B$ produced, and $N$ the point of separation between the compressed and extended parts of the solid, A B CD representing the form of a portion before strain, and $a b \mathrm{OD}$ its form when under the action of the force $\mathrm{F}$.

- Put $\mathrm{G} \mathrm{N}=n, \mathrm{G} \mathrm{B}=k_{0}$, or $\mathrm{N} \mathrm{B}=n+k_{0}, \mathrm{GA}=k_{1}, \mathrm{G} \mathrm{Q}=\mathrm{P}$, and let $\mathrm{A}$ be the area of cross section, and $S$ the compressive force per square inch at the point $B$.

Then for any point $\mathrm{P}$, if $\mathrm{PN}$ be put $=x$, and $y$ be the breadth of the area of cross section of the solid at this point $P$, measured perpendicularly to the plane of the paper, the elementary area of cross section there will be $y d x$, and the force exerted by the portion of the solid to which this elementary area corresponds, will be $\frac{\mathrm{s}}{n+k_{0}} \cdot x \cdot y d x$. The sum or integral of all these forces is equivalent to the force $F$. Hence

$$
\mathbf{F}=\int_{-k_{1}}^{k_{0}} \frac{\mathrm{S}}{n+k_{0}} \cdot x \cdot y d x .
$$

The variable part $x, y d x$ of the second side of this equation expresses the moment of the elementary area with respect to the point $\mathrm{N}$, and since the sum of the moments of the elementary parts of an area is equal to the moment of the whole area applied at its centre of.gravity,

$$
\begin{aligned}
& \int_{-k_{1}}^{k_{0}} x \cdot y d x=\mathrm{A} n \\
& \text { and } \mathrm{F}=\mathrm{SA} \frac{n}{n+k_{0}} .
\end{aligned}
$$

The moment of the force of the elementary area, or its effort to turn the system round $\mathrm{N}$, is $\frac{\mathrm{S}}{n+k_{0}} x, y d x . x$. The sum of these moments is equivalent to the moment of the foree $\mathbf{F}$. Hence 
The value of $\frac{k_{0}}{q}$ depends on the form of cross section of the rib; but there are some leading types of section for which this ratio is readily determined.

(1). For a thin box or I-shaped girder $\left(\frac{k_{0}}{q}\right)^{2}$ may be taken as

$$
\mathrm{F}(p+n)=\int_{-k_{1}}^{\hbar_{0}} \frac{\mathrm{S}}{n+k_{0}} \cdot x^{2} \cdot y d x .
$$

But the variable part $x^{2}, y d x$ is the moment of inertia of the elementary area with respect to the point $\mathbf{N}$, and the integral of this, by a well-known theorem, is equal to $\mathbf{A}\left(q^{2}+n^{2}\right)$, where $g$ is the radius of gyration of the area $\mathbf{A}$ witl respect to its centre of gravity $G$. Hence

$$
\mathbf{F}(p+n)=\frac{\mathrm{S}}{n+k_{0}} \mathbf{A} \cdot\left(q^{2}+n^{2}\right) ;
$$

and dividing this equation by the foregoing, and reducing, there results $p n=q^{2}$. From this it follows that the point $\mathbf{N}$ is so related to the point $Q$, that drawing the line $\mathbf{G} \mathbf{H}$ through $\mathbf{G}$ perpendicular to $\mathbf{A} \mathbf{B}$, and making the length $\mathbf{G} \mathbf{H}=q$, if $I$ and $Q$ be joined, and $H N$ be drawn at right angles to $H Q$, the point $N$, where H $\mathbf{N}$ cuts A B, will be the boundary between the compressed and extended parts of the solid.

The above value of $\mathrm{F}$ gives

$$
\mathbf{S}=\frac{\mathrm{F}}{\mathbf{A}} \cdot \frac{n+k_{0}}{n},
$$

or the total stress $S$ at the outside surface $B$ is equal to the uniform stress $\overline{\mathbf{A}}$ multiplied by the ratio $\frac{\mathrm{NB}}{\overline{\mathrm{NG}}}$. Calling $\mathrm{X}$ the total stress at the point $\mathrm{P}$, and putting $n+z=x$, or $z=\mathrm{G} \mathrm{P}$, then $n+k_{0}: n+z:: \mathrm{S}: \mathrm{X}$. Combining this with the above value of $\mathbf{S}$,

$$
\mathrm{X}=\frac{\mathbf{F}}{\mathbf{A}} \cdot \frac{n+\mathrm{z}}{n}
$$

or the total stress at aliy point is equal to the uniform stress $\frac{\mathbf{F}}{\mathbf{A}}$, multiplied by the ratio $\frac{N P}{N G}$.aking $z=0$, or for the point $G$,

$$
\mathrm{X}=\frac{\mathbf{F}}{\mathrm{A}},
$$

which shows that whatever may be the stresses on the other points the stress at the centre of gravity of the cross section is al ways equal to the force $\mathbf{F}$ divided by the area of cross section, or is the same as if the force $F$ acted in the neutral line $\mathbf{H} \mathrm{G}$.

Substitutiug ${ }_{p}^{\prime 2}$ for $n$ in the above general value of $\mathbf{X}$,

$$
\mathbf{X}=\frac{\mathrm{F}}{\mathbf{A}}\left(\mathbf{1}+\frac{p z}{q^{2}}\right),
$$

and makingr $z=k_{0}$, the result is kiquation $(6 a)$. 
approximately $=1$, and the stress therefore will be $f(1+1)$ or $2 f$; that is, the stress $f$ is doubled at the point where the curve of equilibrium touches the outer surface of the rib, and, writing $-k_{0}$ for $k_{0}$ (see Note to Eq. $\left.(6 a)\right), f(1-1)$, or zero, at the point in the opposite surface.

(2). For a thin circular or elliptical tube, $\frac{k_{0}}{q}$ is nearly $=\sqrt{2 \text {, and }}$ $\left(\frac{k_{0}}{q}\right)^{2}$ nearly $=2$. For this form of section, therefore, the uniform stress $f$ is tripled at the point where the curve of equilibrium coincides with the outer surface of the rib, and there is a force $f(1-2)$ or $-f$, or a tensile force equal to $f$, acting at the other surface of the rib.

(3). For a solid rectangular form of cross section $\frac{k_{0}}{q}$ is equal to $\sqrt{3}$, and $\left(\frac{k_{0}}{q}\right)^{2}=3$, and for this form the uniform compressive force is quadrupled, at the one surface of the rib, and there is a tensile force, of $f(1-3)$ or $2 f$, at the other surface.

(4). For a circular or elliptical solid bar, $\frac{k_{0}}{q}$ is equal to 2 , and $\left(\frac{k_{0}}{q}\right)^{2}=4$. For this form, the uniform compressive force is quintupled at one surface of the rib, and there is a tensile force of $f(1-4)$, or $3 f$, at the other surface.

It will be obvious from these examples that the first-mentioned section is much better fitted for resisting this kind of stress than any of the others.

The general conditions of equilibrium having been explained, the action of vertical forces will be first examined.

\section{Vertical Forces.}

If $P$, Fig. $E$, be any point in an arch rib of which the neutral line is $\mathrm{A} \mathrm{P} \mathrm{C}$, the curve of equilibrium $\mathrm{QS}$, and $\mathrm{P} M$ the perpendicular let fall from $P$ on the direction of the resultant, then the bending moment, as already explained, is

$$
\mathrm{M}=\mathrm{F} \times \mathrm{PM} \text {. }
$$

Draw $\mathrm{PN}$ rertical, cutting the curve of equilibrium in $\mathrm{N}, \mathrm{RN}$ 
tangential at this point $N$, and $P M$ perpendicular to $R N$, also $P R$ horizontal cutting $R N$ in $R$.

Fig. E.

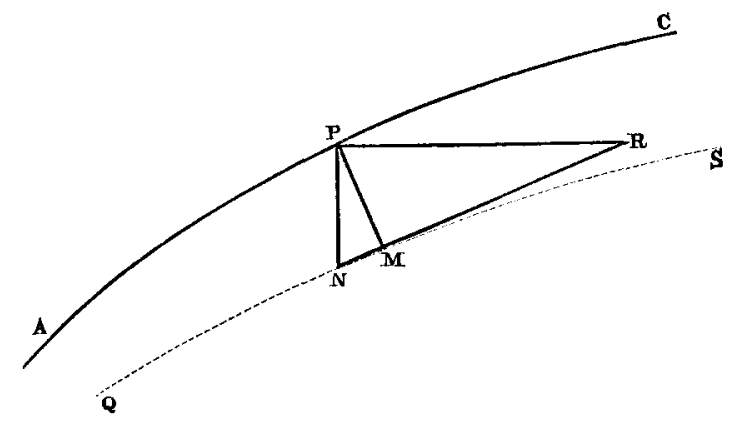

Calling H the horizontal thrust,

$$
\mathbf{F}: \mathrm{H}:: \mathrm{R} \mathbf{N}: \mathrm{R} \mathbf{P} ;
$$

and by similar triangles,

$$
\text { P } \mathbf{M}: \mathbf{R} \mathbf{P}:: \mathbf{P} \mathbf{N}: \mathrm{R} \text { N. }
$$

Combining these proportions,

$$
\mathrm{F} \times \mathbf{P} \mathbf{M}=\mathbf{H} \times \mathbf{P} \mathbf{N} \text {. }
$$

Or, since in the case of vertical pressures the value of $H$ is uniform throughout the curve, the value of $M$ is measured simply by the length of the vertical line $\mathrm{P} N$ between the neutral line of the rib and the curve of equilibrium.

The conditions of equations (3) and (6) are therefore simplified.

If the rib be considered as rigid, and the ends kept from spreading, the condition to be satisfied is (see Fig. F)

$$
\Sigma(P N \times P Q)=0 \text {. }
$$

If the rib is also 'fixed' at $\mathrm{A}$ and $\mathrm{B}$, the conditions are

$$
\begin{aligned}
& \Sigma(P N)=O \\
& \Sigma(P N \times P Q)=0 .
\end{aligned}
$$

The process of construction is therefore as follows (Fig. F) :

Having divided the neutral line of the arch rib into equal portions, draw vertical lines through the centres, $p, p^{\prime}, p^{\prime \prime}$, \&c., of the divisions, which will cut the base line, or line joining A and B, in the points $q, q^{\prime}, q^{\prime \prime}, \&$ c. Also draw an approximate curve of equilibrinm, which will be cut by these verticals in the points $n, n^{\prime} n^{\prime \prime}, \&$ c. 
Fig. F.

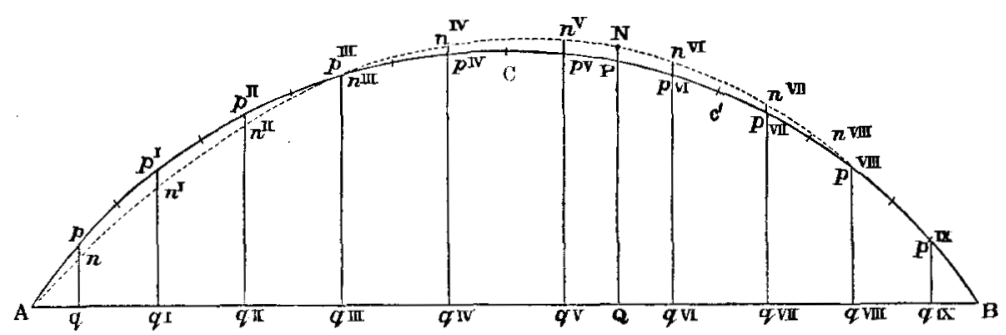

Then, if the rib be considered as rigid, with the points $A$ and $B$ kept from moving sideways, the curve of equilibrium must be such that between the points $\mathrm{A}$ and $\mathrm{B}$,

$$
p n \times p q+p^{\prime} n^{\prime} \times p^{\prime} q^{\prime}+p^{\prime \prime} n^{\prime \prime} \times p^{\prime \prime} q^{\prime \prime}+\& c . \ldots=0 .
$$

That is, those products where the point $n$ is above $p$, as at $n^{w v}, n^{v}, \&$ c., must balance the products where $n$ is below $p$, as at $n, n^{1}, \& c$. By satisfying this condition, the bending moments which increase the curvature and span will exactly neutralize those which diminish the curvature and span, so that the span will be unaltered. It is not difficult to find the correct curve of equilibrium after a few trials. The values of $p n$ and $p q$ can be measured by scale from the diagram, and a sliding rule will be found useful in obtaining the products. In the figure, near the vertex, where the bending moments $p^{\mathrm{iv}} n^{\mathrm{iv}}, \& \mathrm{c}$., are multiplied by the larger lengths $p^{\mathrm{w}} q^{\mathrm{wv}}$, these products are more effective in the summation than at the haunches, where the lengths $p q$ are shorter, and thus the area between the two curves for that part where the neutral line of the rib is above the curve of equilibrium will require to be smaller than for the part where it lies below. ${ }^{1}$

1 Major Browne, R.E., has suggested that, in order to avoid drawing a number of trial curves, the curve of equilibrium may be made to pass accurately through any given point by the use of proportional compasses. Suppose the given point to be $\mathbf{N}^{\prime}$, above or below $\mathbf{N}$, Fee Fig. $\mathbf{F}$; then, having drawn the trial curve which passes through $N$, the curve which passes through $N^{\prime}$ will have the ordinate $Q N$ altered in the ratio $Q N^{\prime}: Q N$; and since any other ordinate, as $q n$, will be altered in the same proportion, this may be done by setting the compasses to the ratio $Q \mathrm{~N}^{\prime}: \mathrm{Q} \mathbf{N}$; or, if straight lines $A \mathrm{~N}, \mathbf{A} \mathbf{N}^{\prime}$ be drawn from $\mathbf{A}$ through $\mathbf{N}$ and $N^{\prime}$, for any two points at the same level, one point on the straight line $A N$, and the other on the trial curve which passes through $\mathrm{N}$, the distance between the lines $\mathbf{A ~ N}$, and $\mathbf{A} \mathbf{N}^{\prime}$, measured vertically, is equal to the distance, also measured vertically, lactween the curve which passes through $\mathrm{N}$, and that which will pass through N'. 
When the arch rib is also fixed in direction at $A$ and $B$, the curve of equilibrium must be so taken that between these points, due regard being had to the signs,

and

$$
p n+p^{\prime} n^{\prime}+p^{\prime \prime} n^{\prime \prime}+p^{\prime \prime \prime} n^{\prime \prime \prime}+\& \mathrm{c} . \ldots=0,
$$

$$
p n \times p q+p^{\prime} n^{\prime} \times p^{\prime} q^{\prime}+p^{\prime \prime} n^{\prime \prime} \times p^{\prime \prime} q^{\prime \prime}+\& c . \ldots=0
$$

Generally, in order to satisfy these two conditions, the springing points of the curve of equilibrium must be different from A and B, and will require to be shifted vertically.

Roughly speaking, the first condition implies that the areas above and below the nentral line of the rib approximately balance one another. A curve being drawn which will nearly satisfy this condition, the curve can then be turned round the points midway between the point of its greatest separation from the neutral line of the rib and the ends $A$ and $B$, until the second condition is also satisfied.

Taking, for example, the circular rib of $120^{\circ}$ examined in Mr. W. Airy's treatise on "Iron Arches," the results from the method now explained may be compared with those arrived at by mathematical investigation.

Fig. 2 (Plate 3 ) represents the rib acted on only by its own weight, considered as uniform per foot run of the rib; Fig. 3, where a load a little less than the weight of the rib, in the proportion of the chord of the are of $60^{\circ}$ to the arc itself, acts at the centre of the crown; and Fig. 4, where this weight acts at $30^{\circ}$ from the crown.

In these figures, the full line represents the line of the arch rib; the dotted line (a) corresponds to the curve of equilibrium of the voussoir arch, or rib hinged at the crown; the dotted line (b) to the rigid arch, with the ends kept from spreading; and the dotted line (c) to the rigid arch, with the ends 'fixed.'

In the following mathematical investigation of the voussoir arch, and arch with the ends fixed, the method used by Mr. W. Airy is adhered to as closely as possible, for the sake of comparison; and a short recapitulation of that method may be made for the better understanding of the case examined, namely, the rigid arch with the ends kept from spreading.

It is only necessary to investigate the case of the weight acting eccentrically, since by making the eccentricity = zero, or the acting weight $=$ zero, this may be made to include the cases of the weight acting on the centre of the crown, or that of the heavy rib acted on by no additional weight. 
Let the radius $\mathrm{OO}=\mathrm{R}$ (Fig. $\mathrm{G}$ ), and $\mathrm{AOC}$, the semi-angle of the $\mathrm{rib},=a$.

Supposing the weight $W$ to act at the point $\mathrm{P}$, let $\mathrm{POC}=\beta$, and let the weight of unity of length of the arch $\mathrm{rib}=\omega$, and let $H$ and $K$ be the horizontal and vertical forces acting at $A, H$ being the horizontal thrust.

For any point $p$ to the right of $\mathrm{P}$, for which the angle $\mathrm{C} \mathrm{O} p=\theta$, the moment $M$ of all the forces acting at $p$ will be-

$\mathbf{M}=$ weight of arc $p \mathbf{A} \times$ horizontal distance of its centre of gravity from $p+\mathrm{W} \times \mathrm{Q} q+\mathrm{H} \times p q-\mathrm{K} \times \mathrm{A} q$.

If $p$ be to the left of $P$, the term $W \times Q q$ in this value of $M$ must be omitted.

To find the first term, $\theta$ being considered as a variable angle, the weight of an elementary portion of the are $R d \theta$ is $\omega \mathbf{R} d \theta$, the horizontal distance of its centre of gravity from $O$ is $R \sin \theta$, and the moment with respect to $\mathrm{C}$ is $\omega \mathbf{R}^{2} \sin \theta d \theta$, the integral of which, for the arc $\mathrm{C} p$ between $\theta=0$ and $\theta=\theta$, is $\omega \mathrm{R}^{2}(1-\cos \theta)$.

Fig. G.

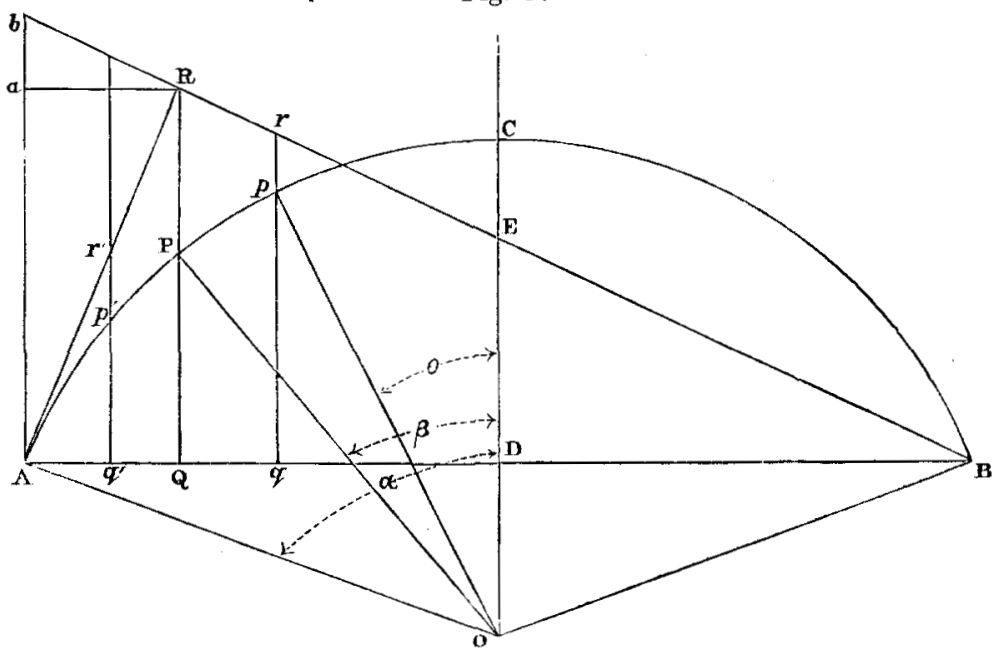

In like manner, the moment of the arc $\mathrm{C} A$ with respect to $\mathrm{C}$ is $\omega R^{2}(1-\cos \alpha)$, and the moment of $p \mathrm{~A}$, or the difference of these two, is $\omega \mathrm{R}^{2}(\cos \theta-\cos a)$.

The horizontal distance of the centre of gravity of $p \mathrm{~A}$ from $\mathrm{C}$ is therefore, $\omega \mathrm{R}(\alpha-\theta)$ being the weight of $p \mathrm{~A}$,

$$
\frac{\omega \mathbf{R}^{2}(\cos \theta-\cos \alpha)}{\omega \mathrm{R}(\alpha-\theta)} \text { or } \mathrm{R} \cdot \frac{\cos \theta-\cos \alpha}{\alpha-\theta}
$$


and its hurizontal distance from $p$ is

$$
\mathbf{R} \cdot \frac{\cos \theta-\cos \alpha}{\alpha-\theta}-\mathrm{R} \sin \theta,
$$

and the moment with respect to $p$,

or,

$$
\omega \mathrm{R}(\alpha-\theta)\left\{\mathrm{R} \frac{\cos \theta-\cos \alpha}{\alpha-\theta}-\mathrm{R} \sin \theta\right\},
$$

Also

$$
\omega \mathrm{R}^{2}(\cos \theta-\cos \alpha+\theta \sin \theta-\alpha \sin \theta) \text {. }
$$

$$
\begin{aligned}
& \mathrm{Q} q=\mathbf{R}(\sin \beta-\sin \theta) \\
& p q=\mathrm{R}(\cos \theta-\cos \alpha) \\
& \mathrm{A} q=\mathrm{R}(\sin \alpha-\sin \theta) .
\end{aligned}
$$

To find the upward pressure $K$, take moments with respect to $\mathrm{B}$ :

$$
\mathrm{K} \times \mathrm{AB}=\mathrm{W} \times \mathrm{QB}+\text { weight of } \mathrm{ACB} \times \mathrm{DB},
$$

or, $\quad \mathrm{KR}(2 \sin \alpha)=\mathrm{WR}(\sin \alpha+\sin \beta)+\omega \mathrm{R} 2 \alpha \mathrm{R} \sin \alpha$

$$
\mathrm{K}=\frac{\mathrm{W}}{2} \cdot \frac{\sin \alpha+\sin \beta}{\sin \alpha}+\omega \mathrm{R} \alpha,
$$

and the above value of $\mathrm{M}$ becomes, after reduction, as found by Mr. Airy,

$$
\begin{aligned}
\mathrm{M}= & \omega \mathrm{R}^{2}(\cos \theta+\theta \sin \theta-\cos \alpha-\alpha \sin \alpha) \\
& +\mathrm{W} \mathrm{R}(\sin \beta-\sin \theta) \\
& +\mathrm{HR}(\cos \theta-\cos \alpha) . . . . \\
& +\frac{\mathrm{W}}{2} \mathrm{R} \cdot \frac{\sin \alpha+\sin \beta}{\sin \alpha}(\sin \theta-\sin \alpha),
\end{aligned}
$$

where it is to be observed that for a point to the left of $P$, the term $\mathrm{W} \times \mathrm{Q} q$, or the term $\mathrm{WR}(\sin \beta-\sin \theta)$, must be omitted between $\theta=+\beta$ and $\theta=+\alpha$.

From the manner in which this value of $\mathbf{M}$ has been obtained, it will evidently have the same expression, whether the arch is considered as a voussoir arch, or as a rigid arch with the ends kept from spreading, the difference between these two cases being that the value of $\mathbf{H}$ is different for each.

For the rigid arch with the ends fixed, there will be a bending rnoment $\mu$, for the present unknown, acting at $A$, and over the whele arch rib, until it is met and counteracted by the equal but opposite moment $-\mu$ acting at the point B. In this cnse, therefore, the bending moment at any point will be $M+\mu$ instead of $\mathrm{M}$. 
The unknown horizontal thrust $H$, which appears in the value of $\mathbf{M}$, will now be determined.

For the voussoir arch, there being no bending moment or transverse stress at the crown, taking moments to the left of $\mathrm{C}$ with respect to that point:

Moment of are $\mathrm{CA}+\mathrm{W} \times \mathrm{QD}+\mathrm{H} \times \mathrm{DC}=\mathrm{K} \times \mathrm{AD}$, or,

$$
\begin{gathered}
\omega \mathbf{R}^{2}(1-\cos \alpha)+\mathrm{W} \times \mathrm{R} \sin \beta+\mathrm{HR}(1-\cos \alpha) \\
=\left\{\frac{\mathrm{W}}{2} \frac{\sin \alpha+\sin \beta}{\sin \alpha}+\omega \mathrm{R} \alpha\right\} \mathrm{R} \sin \alpha,
\end{gathered}
$$

and reducing,

$$
\mathrm{H}=\frac{\mathrm{W}}{2} \cdot \frac{\sin \alpha-\sin \beta}{1-\cos \alpha}+\omega \mathrm{R}\left(\frac{\alpha \sin \alpha}{1-\cos \alpha}-1\right)
$$

In the case of the rigid arch with the ends kept from spreading, the condition of equation (6) must be satisfied, or

and since

$$
\mathbf{\Sigma}(\mathbf{M} \times \mathbf{P Q})=\mathrm{O}
$$

$$
\begin{gathered}
\Delta s=\mathrm{R} \Delta \theta \\
\int_{-a}^{+a} \mathrm{M} \cdot \mathrm{R}(\cos \theta-\cos a) \mathrm{R} d \theta=0 .
\end{gathered}
$$

Bearing in mind the omission of the term WR $(\sin \beta-\sin \theta)$ between $+\beta$ and $+\alpha$ in the integration, the resulting value of $\mathrm{H}$, as given in Mr. Airy's treatise, is

$$
\mathrm{H}=\omega \mathrm{R} \frac{\alpha^{2} \sin \alpha \cos \alpha+\alpha\left(\frac{9}{4} \cos ^{2} \alpha-\frac{1}{4} \sin ^{2} \alpha\right)-\frac{9}{4} \sin \alpha \cos \alpha}{\frac{3}{2} \sin \alpha \cos \alpha-\alpha\left(\frac{1}{2} \sin ^{2} \alpha+\frac{3}{2} \cos ^{2} \alpha\right)}
$$

$+\frac{W}{2} \cdot \frac{\frac{3}{2} \cos ^{2} \alpha-\frac{1}{2} \cos ^{2} \beta-\cos \alpha \cos \beta+(\alpha \sin \alpha-\beta \sin \beta) \cos \alpha}{\frac{3}{2} \sin \alpha \cos \alpha-\alpha\left(\frac{1}{2} \sin ^{2} \alpha+\frac{3}{2} \cos ^{2} \alpha\right)}$.

For the rigid arch with the ends fixed, both in direction and position, $\mathbf{M}+\mu$ must be substituted for $\mathbf{M}$ in the above integration, which changes it to

$$
\int_{-a}^{+a} \mathrm{M}(\cos \theta-\cos \alpha) d \theta+\int^{+a} \mu(\cos \theta-\cos \alpha) d \theta=0,
$$

Integrating,

$$
\begin{aligned}
& O=\omega \mathbf{R}^{2}\left\{\begin{array}{c}
\left.-\frac{9}{2} \sin \alpha \cos \alpha+\alpha\left(\frac{9}{2} \cos ^{2} \alpha-\frac{1}{2} \sin ^{2} \alpha\right)\right\} \\
+\alpha^{2} \cdot 2 \sin \alpha \cos \alpha
\end{array}\right\} \\
& +\mathrm{WR}\left\{\begin{array}{c}
\frac{3}{2} \cos ^{2} \alpha-\frac{1}{2} \cos ^{2} \beta-\cos \alpha \cos \beta \\
+(\alpha \sin \alpha-\beta \sin \beta) \cos \alpha
\end{array}\right\} \\
& +\mathrm{HR}\left\{a\left(\sin ^{2} \alpha+3 \cos ^{2} \alpha\right)-3 \sin \alpha \cos \alpha\right\} \\
& \quad+\mu \cdot 2(\sin \alpha-\alpha \cos \alpha) .
\end{aligned}
$$


In this case the condition of equation (3) is also to be satisfied,

or,

$$
\int_{-a}^{+a} \operatorname{Mr} d \theta=0
$$

Substituting in this the value of $M$ from equation (7), integrating between the limits $-\alpha$ and $+\alpha$, omitting the term $W R$ $(\sin \beta-\sin \theta)$ between $+\beta$ and $+\alpha$, and reducing,

$$
\begin{aligned}
\mathrm{O} & =\omega \mathrm{R}^{2}\left\{2\left(\frac{\sin \alpha}{\alpha}-\cos \alpha\right)-\alpha \sin \alpha\right\} \\
& +\frac{\mathrm{W}}{2} \mathrm{R}\left\{\frac{\cos \beta}{\alpha}-\frac{\cos a}{\alpha}+\frac{\beta}{\alpha} \sin \beta-\sin \alpha\right\} \\
& +\mathrm{HR}\left\{\frac{\sin \alpha}{\alpha}-\cos \alpha\right\} \\
& +\mu .
\end{aligned}
$$

From (8) and (9) the values of $H$ being determined and substituted in (7), the bending moment MI at any point of the voussoir and rigid arch with the ends kept from spreading may be found; and in like manner from (10) and (11) the values of $H$ and $\mu$ may be determined, and the resulting value of $M+\mu$ from (7), will give the bending moment at any point of the rigid areh with the ends fixed.

For $\alpha=60^{\circ} \quad \beta=0^{\circ}$.

Equation (8) becomes

and equation (9),

$$
\Pi=.866 \mathrm{~W}+.814 \omega R,
$$

$$
\mathrm{H}=\cdot 631 \mathrm{~W}+\cdot 787 \omega \mathrm{R} ;
$$

also equations (10) and (11) become

$$
\begin{aligned}
& \mathrm{O}=-\cdot 172 \mathrm{WR}-\cdot 213 \omega \mathrm{R}^{2}+\cdot 272 \mathrm{II} \mathrm{R}+\cdot 685 \mu \\
& O=-.195 \mathrm{WR}-.253 \omega \mathrm{R}^{2}+\cdot 327 \mathrm{H} \mathrm{R}+\mu,
\end{aligned}
$$

giving

$$
\begin{aligned}
& \mathrm{H}=\cdot 805 \mathrm{~W}+\cdot 841 \omega \mathrm{R} \\
& \mu=-.022 \omega \mathrm{R}^{2}-.069 \mathrm{WR} .
\end{aligned}
$$

In like manner, for $\alpha=60^{\circ}, \beta=30^{\circ}$, equation (8) lecomes

$$
H=-366 \mathrm{~W}+\cdot 814 \omega \mathrm{R},
$$

and equation (9),

$$
\mathrm{H}=\cdot 406 \mathrm{~W}+\cdot 787 \omega \mathrm{R} ;
$$


Equations (10) and (11) become

$$
\begin{gathered}
\mathrm{O}=-\cdot 110 \mathrm{WR}-\cdot 213 \omega \mathrm{R}^{2}+\cdot 272 \mathrm{HR}+\cdot 685 \mu \\
\mathrm{O}=-\cdot 133 \mathrm{WR}-\cdot 253 \omega \mathrm{R}^{2}+\cdot 327 \mathrm{HR}+\mu \\
\mathrm{H}=\cdot 401 \mathrm{~W}+\cdot 841 \omega \mathrm{R} \\
\mu=-.022 \omega \mathrm{R}^{2}+\cdot 002 \mathrm{WR} .
\end{gathered}
$$

giving

To compare the bending moments with those derived from Figs. 2, 3, and 4, sinco

$$
\begin{gathered}
\mathrm{M}=\mathrm{H} \times \mathrm{PN} \text { (Fig. F) } \\
\mathrm{PN}=\frac{\mathrm{M}}{\mathrm{H}},
\end{gathered}
$$

or, in the case of the arch with the ends fixed in direction,

$$
\mathrm{PN}=\frac{\mathrm{M}+\mu}{\mathrm{H}} \text {. }
$$

Now, if the values of $\mathrm{P} \mathrm{N}$ be calculated from these equations, it will be found that they can, for all practical purposes, be equally well ascertained by scaling their equivalents $\mathrm{P} \mathrm{N}$ from Figs. 2, 3 , and 4 (Plate 3).

Before parting with the above formulæ, an examination of them shows that the expressions for $\mathbf{H}$ and $\mathbf{M}$, or $\mathbf{M}+\mu$, are the algebraical sums of two parts, one corresponding to $\omega=O$, and the other to $\mathrm{W}=\mathrm{O}$.

It follows from this, that if at any point the stress, which is proportional to the bending moment, caused by the weight of the rib itself, or rib and its fixed loading, be found, and also that caused by a moving load acting on the point, the total stress is obtained by adding these two stresses together with their proper signs.

As the action of a moving load can thus be separated from the action of a fixed load, it may be useful to examine the cases of a circular rib of $120^{\circ}$, and of a semi-circular arch rib.

\section{Action of the Weight of the Arch.}

Figs. 2 and 5 (Plate 3 ) show the stresses arising from the weight of the arch itself considered as the fixed load. The vertex and springing points were determined by the above formulæ, and the curves of equilibrium drawn to pass through these points. Calling $P$ the weight of the semi-arch, and $R$ its radius, the values of $H$, 
$\frac{\mathrm{M}}{\mathrm{H}}$ when $\theta=0$, and of $\frac{\mu}{\mathrm{H}}$ are given in the following table:

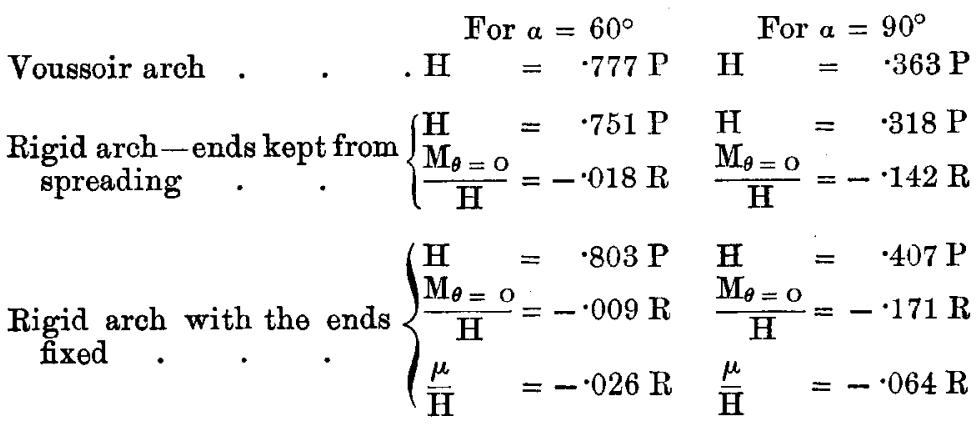

By these values of $\frac{M}{\bar{H}}$ when $\theta=0$, and of $\frac{\mu}{H}$, the vertices and springing points of the curves were determined. It will be observed that the stresses on the rigid arch are in both cases reduced to about one-half by fixing the ends.

The curves of equilibrium for a moving load will now be considered.

\section{Action of a Moving Load.}

If the load on a particular point of the arch be the only force acting, since there are then no forces to bend the curve of equilibrium, it becomes two straight lines, meeting in an apex vertically above the load. For the voussoir areh and rigid arch of invariable span, these lines pass through the centres of the ends of the arch rib.

That the bending moments are measured by the vertical ordinates between these lines of equilibrium and the curve of the rib may be shown as follows:

Putting $\omega=\mathrm{O}$ in equation (7), the value of $\frac{M}{\bar{H}}$ after reduction is

$\overline{\mathbf{H}}=\mathbf{R}\left\{\cos \theta-\cos \alpha-\frac{1}{2} \frac{\mathrm{W}}{\mathrm{H}} \cdot \frac{\sin \alpha+\sin \theta}{\sin \alpha}(\sin \alpha-\sin \beta)\right\}$

If $P$ (Fig. G) be the position of the weight, draw the vertical lines $\mathrm{QP} \mathrm{R}$ and $\mathrm{A} b$ through $\mathrm{P}$ and $\mathrm{A}$, and the horizontal line $\mathrm{R} a$ through $R$, an assumed apex or intersection of the lines of equilibrium R A, R B. 
Then

But

Therefore

Also

or,

and

$$
\mathrm{W}: \mathrm{H}:: \mathrm{A} b: a \mathrm{R} \text {. }
$$

$$
a \mathrm{R}=\mathrm{QA}=\mathrm{R}(\sin \alpha-\sin \beta) \text {. }
$$

$$
\mathrm{A} b=\mathrm{R} \frac{\mathrm{W}}{\mathrm{H}}(\sin \alpha-\sin \beta) \text {. }
$$

$\mathrm{B} D: \mathrm{ED}:: \mathrm{B} \mathrm{Q}: \mathrm{Q} R$,

$$
\begin{aligned}
\mathrm{QR} & =\mathrm{ED} \cdot \frac{\mathrm{B} Q}{\mathrm{BD}}=\mathrm{ED} \cdot \frac{\mathrm{R}(\sin \alpha+\sin \beta)}{\mathrm{R} \sin \alpha} \\
& =\frac{\mathrm{R}}{2} \frac{\mathrm{W}}{\mathrm{H}}(\sin \alpha-\sin \beta) \frac{\sin \alpha+\sin \beta}{\sin \alpha}
\end{aligned}
$$

$$
\begin{gathered}
\mathrm{PR}=\mathrm{QR}-\mathrm{QP} \\
=\frac{\mathrm{R}}{2} \overline{\mathrm{H}} \cdot \frac{\sin \alpha+\sin \beta}{\sin \alpha}(\sin \alpha-\sin \beta)-\mathrm{R}(\cos \beta-\cos \alpha) ;
\end{gathered}
$$

the same as the above value of $\frac{\mathrm{M}}{\mathrm{H}}$ when $\theta=\beta$, but with an opposite sign, since $M$ ' has been treated as negative when $R$ is above $P$. Then if $p$ be a point to the right of $\mathrm{P}$, for which the angle $p \mathrm{OC}=\theta$,

and

$$
\mathrm{B} \mathrm{Q}: \mathrm{B} q:: \mathrm{QR}: q r,
$$

$$
\begin{aligned}
q r=\mathrm{Q} R & \frac{\mathrm{B} q}{\mathrm{BQ}}=\frac{1}{2} \frac{\mathrm{W}}{\mathrm{H}} \mathrm{R} \frac{\sin \alpha+\sin \beta}{\sin \alpha}(\sin \alpha-\sin \beta) \frac{\sin \alpha+\sin \theta}{\sin \alpha+\sin \beta} \\
& =\frac{1}{2} \frac{\mathrm{W}}{\mathrm{H}} \cdot \mathrm{R} \frac{\sin \alpha+\sin \theta}{\sin a}(\sin \alpha-\sin \beta),
\end{aligned}
$$

and

$$
p r=q r-q p
$$

$$
=\frac{1}{2} \frac{W}{H} \mathbf{R} \cdot \frac{\sin a+\sin \theta}{\sin a}(\sin a-\sin \beta)-\mathbf{R}(\cos \theta-\cos \alpha) ;
$$

the same as the general value of $\frac{\mathbf{M}}{\mathbf{H}}$ in (12), but with a negative sign, as already explained.

For a point to the left of $P$, the term $W R(\sin \beta-\sin \theta)$ being omitted in the value of $\mathbf{M}$, equation (7) gives

$$
\frac{\mathrm{M}}{\mathrm{H}}=\mathrm{R}(\cos \theta-\cos \alpha)+\frac{1}{2} \frac{\mathrm{W}}{\mathrm{H}} \mathrm{R} \frac{\sin \alpha+\sin \beta}{\sin \alpha}(\sin \theta-\sin a),
$$

and from Fig. G,

$$
\begin{gathered}
\mathrm{A} \mathrm{Q}: \mathrm{A} q^{\prime}:: \mathrm{QR}: q^{\prime} r^{\prime} \\
q^{\prime} r^{\prime}=\mathrm{QR} \frac{\mathrm{A} q^{\prime}}{\mathrm{AQ}}=\mathrm{Q} \mathrm{R} \cdot \frac{\sin \alpha-\sin \theta}{\sin \alpha-\sin \beta} \\
=\frac{1}{2} \frac{\mathrm{W}}{\mathrm{II}} \mathrm{R} \cdot \frac{\sin \alpha+\sin \beta}{\sin \alpha}(\sin \alpha-\sin \theta) .
\end{gathered}
$$


and

$$
p^{\prime} r^{\prime}=q^{\prime} r^{\prime}-q^{\prime} p^{\prime}
$$

$$
=\frac{1}{2} \frac{W}{\mathbf{H}} \mathrm{R} \cdot \frac{\sin \alpha+\sin \beta}{\sin \alpha}(\sin \alpha-\sin \theta)-\mathrm{R}(\cos \theta-\cos \alpha),
$$

the same as the above value of $\frac{M}{\mathbf{H}}$, but with the negative sign.

For the voussoir arch, since there is no bending moment at the crown, one of the lines of equilibrium is fixed by the condition that it must pass through the crown, and through the end of the arch farthest from the load; the other line is fixed by making it pass through the end of the arch nearest the load, and tho point where a vertical line through the load cuts the first-mentioned line.

For the rigid arch, a curve line above the arch can be found, such that, for any given position of the load, the vertical line through the load will intersect this curve in a point, which point will be the apex of the two lines of equilibrium for that position of the load.

To draw this curve, or locus of the apex, it is only necessary to find from (12) the value of $\frac{M}{H}$ when $\theta=\beta$, and lay off above the point where the weight acts a vertical length equal to this. By finding a sufficient number of points the curve can be drawn.

Figs. 7 to 10 (Plate 3) show the lines of equilibrium, and the locus of the apex for the arc of $120^{\circ}$, and for the semicircular are. For the latter, the locus becomes a straight line, drawn at a distance of $\frac{\pi}{2} \mathrm{R}$ above the centre of the circle, or its height above this centre is equal to the length of the quadrant.

On Figs. 8 and 11, for the rigid arch with the ends fixed, the lines of equilibrium are shown for different positions of the load, the springing points of these lines being determined by the values of $\frac{\mu}{\mathrm{H}} \cdot$ from (10) and (11).

Where so many lines were to be found, it was easier to fix their points of intersection by formulæ already obtained than to find each by a separate graphic construction. The lines are in the same positions as would have been found by construction, the lines of equilibrium on Figs. 7 and 10 satisfying Eq. (6), and those on Figs. 8 and 11, Equations (3) and (6).

But the lines on these Figs. only serve to compare among themselves the stresses on different points of the rib for the same position of the load. The bonding moment being compounded of the horizontal thrust, as well as the height P N (Fig. F), it is 
necessary, in order to compare the absolute stresses for different positions of the load, to take into account the change of horizontal thrust. This comparison may be made by arranging that all the stresses may contain a common factor, and the simplest common factor is the weight itself. The bending moment, or $\mathrm{H} \times \mathrm{PN}$, may then be assumed equal to $W \times x$, giving,

$$
x=\frac{\mathrm{H}}{\mathrm{W}} \cdot \mathrm{PN} \text {. }
$$

That is, by plotting at the different points of the rib, heights or depths less than $\mathrm{PN}$ in the ratio of $\mathrm{H}$ to $\mathrm{W}$, these new heights being multiplied by $\mathrm{W}$, will give the bending moments.

In this manner, the network of lines on Fig. 6 has been drawn, and the dotted lines, which are passed through the points of maximum bending moment, show the greatest stresses to which the rib is exposed during the passage of a load from one side to the other of the arch rib. It will be observed that the greatest stress occurs when the load is about midway between the orown of the arch and the abutment.

On Figs. 7 to 11, only the dotted lines of maximum bending moment have been drawn. This maximum bending moment is equal to the weight multiplied by the vertical distance between the neutral line of the arch rib, and the dotted line, to the same scale as the arch rib is drawn. For example, in a semicircular rigid arch, of 100 feet span, with the ends kept from spreading, the greatest bending moment for a weight of 10 tons passing along it occurs when the weight is over the centre of the rib, and is measured by 10 tons $\times 9$ feet tending to lessen the curvature; and during the passage of the load there is at a point $50^{\circ}$ from the crown a bending moment of 10 tons $\times 5$ feet 3 inches tending to increase the curvature.

Putting $k$ for the length of the line P N (Fig. F),

$$
\mathrm{M}=\mathrm{H} k,
$$

and denoting by $\mathrm{M}^{\prime}, \mathrm{H}^{\prime}$, and $k^{\prime}$, similar quantities corresponding to a weight $W^{\prime}$, in like manner,

$$
\mathbf{M}^{\prime}=\mathbf{H}^{\prime} k^{\prime}
$$

and $\quad \mathbf{M}+\mathbf{M}^{\prime}=\mathrm{H} k+\mathrm{H}^{\prime} k^{\prime}$.

But the horizontal thrust for $W$ and $W^{\prime}$ acting together will be II $+\mathrm{H}^{\prime}$. Let, therefore,

$$
\left(\mathbf{H}+\mathbf{H}^{\prime}\right) k^{\prime \prime}=\mathbf{H} k+\mathbf{H}^{\prime} k^{\prime} .
$$

I'llece

$$
k^{\prime \prime}=k \cdot \frac{\mathrm{II}}{\mathbf{H}+\mathbf{H}^{\prime}}+k^{\prime} \cdot \frac{\mathbf{H}^{\prime}}{\mathbf{H}+\mathbf{H}^{\prime}} . .
$$


or the length $\mathrm{PN}$ for the combined curve may be found by adding together the lengths for $\mathrm{W}$ and $\mathrm{W}^{\prime}$, each reduced in the ratio of its horizontal thrust to the total horizontal thrust. An example of this is given in the voussoir arch first examined.

\section{Action of a uniformly distributed Load.}

Since the values of $H$ and $M$ are proportional to $W$, the stress caused by any number of weights is the sum of the stresses caused by each separately, and Figs. 6 to 11 give the means of ascertaining the position and amount of greatest stress for a uniform load, supposed to be put on the arch beginning from one end, until the whole is covered.

But the action of a uniform load on a definite part or the whole of an arch can be arrived at with greater facility by the constructive method of drawing the curves of equilibrium. Examples of this for the rigid arch, with the ends kept from spreading, are given on Figs. 12, 13 and 14, Plate 3. Fig. 12 represents an arch whose rise is $\frac{1}{10}$ th of the span. One of the dotted lines $(b)$ shows the curve of equilibrium when a moving load, equal per foot run to $\frac{1}{2}$ the fixed load, has a uniform horizontal distribution over one-half of the arch in its central part; and the other dotted line (c) shows the curve when the uniform horizontal distribution of the moving load is over the half of the arch from the centre to the abutment.

Fig. 13 represents the arch of $120^{\circ}$ already examined. The dotted line (a) is the curve, a parabola, for the rigid arch with the span invariable, under a uniform horizontal loading all over. The dotted line (b) shows the curve when a moving load, equal to $\frac{1}{2}$ the fixed load per foot run, extends over $\frac{1}{4}$ of the span each way from the centre, or over $\frac{1}{2}$ the span altogether, the dotted line (c), when the same moving load extends from the centre to one of the abutments.

For the sake of comparison with the last, Fig. 14 shows the curves of the rigid arch of invariable span, the form of the arch being not circular but parabolic. The parabola is the curve of equilibrium for a uniform horizontal load, so that when the loading is distributed all over the span, the curve of equilibrium coincides with the curve of the arch rib, and there is no transverse stress. The dotted line $(b)$ shows the curve with the abovementioned moving load extending over $\frac{1}{4}$ th of the span each way from the centre; the dotted line (c) shows the curve when the moving load extends from the centre to one of the abutments. 
The loading being supposed uniform horizontally, the weight which acts at each of the verticals is proportional to the horizontal projection of the division of the arc which it bisects. From these weights the curves of equilibrium have been constructed so as to satisfy equation (6).

It will be observed that when the moving load extends from the centre to the abutment, although the transverse stress tending to diminish the curvature of the arch at its loaded part is considerable, there is an equal, and in the case of the arc of $120^{\circ}$ a rather greater, transverse stress at the unloaded haunch tending to increase the curvature. Since the inequality of the loading may act on the opposite side of the arch, the curves may be transferred from one side to the other, and the stresses reversed. When the moving load acts on $\frac{1}{4}$ of the span each way from the centre, the transverse stress tending to increase the curvature at the haunches is rather greater than the stress of the opposite kind at the centre of the arch.

The curves for the parabolic rib show rather less stresses for partial loading than those for the circular rib, so that the former, which has no transverse stress when the load is uniformly distributed over it horizontally, has a slight advantage over the circular rib in the event of unequal loading. As the rise of the arch is diminished, the circular and parabolic curves come so close together as to be practically the same.

\section{Siraight or Curved Girder.}

Putting $m$ for the bending moment of all the vertical forces, the value of $\mathrm{M}$ in Eq. (7)-Fig. G-may be written

$$
\mathbf{M}=m+\mathrm{H} \cdot \mathrm{PQ} \text {. }
$$

This quantity $m$, or the sum of all those terms which do not contain $\mathrm{H}$, is nothing else than the bending moment, supposing the arch were a girder.

If the arch becomes a girder, and

$$
\mathbf{M}=m \text {, }
$$

then

$$
\mathrm{H}=\mathrm{O} \text {; }
$$

and, as already remarked, the ordinates of the curve of equilibrium are infinite.

But (Fig. $F$ ) bearing in mind that $M$ is negative at the point $P$,

and

$$
\begin{gathered}
\mathbf{M}=-\mathbf{H} \cdot \mathbf{P N}=m+\mathbf{H} \cdot \mathbf{P Q}, \\
-m=\mathrm{H}(\mathrm{PQ}+\mathrm{PN})=\mathbf{H} \cdot \mathbf{X} \mathbf{Q} .
\end{gathered}
$$


'That is, $\mathrm{H} \cdot \mathrm{NQ}$ is the graphic representation of the bending moment of a girder of the same shape and loading as the arch. Since N Q is the vertical distance between the curve of equilibrium and the straight line, the bending moment is independent of the shape of the arch, which may be either curved or straight.

A straight or curved girder then may be considered as an arch of any rise, but without horizontal thrust; and if its length be divided into parts, and any curve of equilibrium drawn for the weights, continuous or discontinuous, acting on these parts, the stress at any point $\mathrm{P}$ will be represented by the value of $\mathrm{H} \cdot \mathrm{N} Q$. Since $H$ varies inversely as $\mathrm{N} Q, \mathrm{H} \cdot \mathrm{N} \mathrm{Q}$ will be the same for all the curves of equilibrium that can be drawn. This remark leads to the following method of ascertaining the stresses on a continuous beam.

\section{Continuous Beam.}

While examining, in the year 1849 , the stresses on continuous beams for the late Mr. Brunel, in reference to the large bridge at Chepstow, the lower girder of which is virtually a continuous beam of five unequal spans, the Author, using the method of Navier, found the subject one of no inconsiderable difficulty, from the number and complexity of the eliminations required; and he was gratified by the formulæ at which he then arrived, for beams up to five spans, being completely verified by the experimental tests to which he subjected them, and which were devised by Mr. Brunel. One of these experiments is described in Mr. Edwin Clark's work on the Britannia Bridge. ${ }^{1}$

Since that time, the discovery of the "theorem of three moments" has introduced great simplicity in the consideration of continuous beams of uniform section; and recently Mr. Heppel, in a Paper read before the Royal Society of London, ${ }^{3}$ has been the first to solve the general problem of the continuous beam of varying section, and of any number of spans or manner of loading. The solution depends on certain definite integrals, which if they cannot always be obtained in a finite form, may, by the method of quadratures, be approximated to with as great numerical accuracy as may be desired.

Aware of the difficulty of the subject, the Author ventures to present it under a somewhat different aspect, and hopes to show,

1 Vide Britannia and Conway 'Tubular Bridges, vol. i., p. 462.

2 Vide Minutes of Proceelings Inst. O.E., vol. xxix., pp. 44-48.

${ }^{3}$ Vide Proceedings of the Royal Society of London, vol. xix., p. 56. 
in the following remarks, that the stresses on a continuous beam of varying or uniform section may be ascertained with sufficient accuracy for practical purposes by a modification of the method of finding the stresses on rigid arches.

It will be convenient in the first instance to consider beams of uniform section.

For these, though loaded in any manner whatever, if they are supposed disconnected at the piers, a curve of cquilibrium for each span may be drawn, the rise of the curve being arbitrary. And all the forces being vertical, the bending moment or stress at any point (see p. 38) would be measured by the horizontal thrust, multiplied by the vertical ordinate.

The condition that the sum of the upward pressures at the points of support must be equal to the downward pressures of the weights, would be complied with, since the weight acting on each span would be borne by the end supports.

When the beams are supposed to be connected together, so as to form one continuous beam extending over the whole of the spans, the above condition must still be satisfied; but, in consequence of the continuity, the weights borne by the different points of support will not be the same as when the beams are unconnected, and the differences will introduce bending moments at the points of support, and at every point of the beam, except at the two ends, where the bending moments are zero, unless external forces act on the beam at these points.

On the diagrams of the curves of equilibrium, the bending moments at the piers may be represented by vertical straight lines, each being the bending moment divided by the horizontal thrust.

On Fig. H, Plate $3_{A}, \mathrm{ACB}$ and $\mathrm{BED}$ being curves of equilibrium, for the weights acting on the spans $A B$, and $B D, B x$ and $D y$ represent the bending moments at $B$ and $D$, and the stress at any point $t$, due to the alteration of pressure at the points of support caused by the continuity, will be represented by a vertical ordinate $t s$, from the straight line A BD to the straight lines $\mathrm{A} x, x y$; and the actual stresses at any point $t$ of the beam supposed continuous is represented by the difference between the ordinate of the curve and of the straight line, and is therefore equal to the horizontal thrust multiplied by $r s$.

And because, for any one pier, the bending moment there must be the same, whether moments are reckoned to the right or left, the horizontal thrust on one side, which requires to be multiplied by the length of the vertical line already mentioned to give the bending moment, must be equal to the horizontal thrust 
on the other side; that is, the horizontal thrusts of two adjoining curves must be the same; and since the thrust is also constant throughout the same curve, it follows that in order to have a set of curves, the ordinates of which will represent the stresses on a continuous beam, it is necessary that the horizontal thrusts for the different spans should be all equal to one another, or the arches which these curres represent should be balanced arches. One curve being drawn, all the others are determined by this condition.

Commencing at one end $\mathrm{A}$ of the beam (Fig. $\mathrm{H}$ ), draw the curve of equilibrium $\mathrm{ACB}$ for the weights acting on the span $\mathrm{AB}$, of any convenient rise $\mathrm{CG}$, measured on the same scale as that for the span $\mathrm{A} \mathrm{B}$, and call $\mathrm{H}$ the horizontal thrust of this curve.

To draw the curve for the next span, dividing the length BD in the middle, or any other convenient point $m$, let $\mathrm{PP}^{\prime}$ and $\mathrm{Q}^{\prime}$ be the vertical lines passing through the centres of gravity of the sums $\mathrm{P}$ and $\mathrm{Q}$ of the weights which act between $m$ and $\mathrm{B}$ and $m$ and $\mathrm{D}$. From the positions of these weights, the vertical reactions at $B$ and $D$ may be found, the beam being at present not supposed to be continuous. Making $\mathrm{B} a$ equal to $\mathrm{H}$, and $a b$ equal to the upward reaction at $B$, the line $B b$ will be tangential to the curve of equilibrium at the point $\mathrm{B}$, and will cut the vertical line $P \mathrm{P}^{\prime}$ in the point $P^{\prime}$. Similarly, the tangent at $D$ may be found, and this will cut the vertical line $Q^{\prime}$ in the point $Q^{\prime}$. Joining $P^{\prime}$ and $Q^{\prime}$, the line $\mathrm{P}^{\prime} \mathrm{Q}^{\prime}$ will be tangential to the curve of equilibrium at the point $\mathrm{E}$ in the vertical line $\mathrm{E} m$, and the rise of the curve $\mathrm{B} \mathrm{ED}$ will thus be determined. The curve for the span BD may then be drawn, and so on for the other spans.

Having thus drawn, on the horizontal line passing through the points of support, a series of curves of uniform horizontal thrust for the different spans, divide the whole length of the beam into any convenient number of equal parts, and draw vertical lines $t_{0} r_{0}, t_{1} r_{1}$, \&c., through the centres of these divisions. If $\mathrm{B} x, \mathrm{D} y$, $\& c$. , be drawn tentatively to represent the bending moments at B, D, \&c., caused by the altered pressures on the piers, the ordinates $s_{0} r_{0}, s_{1} r_{1}$, \&c., will represent tentatively the stresses on the beam. And if a curve $A_{K_{0}} B_{1} K_{1} D_{1} F_{1}$, \&c., be drawn in such manner that at any point $t$ the ordinate $t u$ is the algebraical sum, to any convenient scale, of all the ordinates $r_{0} s_{0}, r_{1} s_{1}$, \&c., from $\mathrm{A}$ up to $t, t u$ will represent the area $\mathrm{A} \mathrm{C} r \&$, or the change of angle of the elastic curve of the beam between $\mathrm{A}$ and $t$. Drawing, then, through this curve, and for the first span, a line $A_{0} B_{i}$, parallel to $A B$, so as to make the area $A A_{0} K_{0}$ equal to the 
area $B_{1} B_{0} K_{0}$, the line $A A_{0}$ will represent the constant to be added in the integration to make these areas equal, or the ordinate of the elastic curve equal to zero at the point $B$; since this ordinate is represented by the sums of the ordinates or the area of this derived curve. By the addition of the constant, the axis is shifted from $A B$ to $A_{0} B_{0}$; and since the slope of the beam at the point $B$ for the part $A B$ must be the same as for the part $B D$, the curve $B_{1} K_{1} D_{1}$ for the second span must have the same ordinate $\mathrm{B}_{0} \mathrm{~B}_{1}$ at $\mathbf{B}$ as that of the curve of the first span. That is, the axis of the curve for the second span lies in the prolongation of the axis for the first span. The same condition of equality of areas subsists, or the area $B_{1} B_{0} K_{1}$ must be equal to the area $\mathrm{D}_{0} \mathrm{D}_{1} \mathrm{~K}_{1}$. Similar remarks apply to the remaining spans.

In order, therefore, to ascertain the stresses on a continuous beam of uniform section, the primary curves of equilibrium must have the same horizontal thrust, and the derived curve $A K_{0} B_{1} K_{1}$, \&c., must be capable of being cut by a line parallel to $A B$, in such manner, that the areas above and below this line, for each span, shall balance one another. 'The heights $\mathrm{B} x, \mathrm{D} y$, are to be arranged so that this condition is satisfied.

Fig. H, Plate $3 \mathrm{~A}$, is an example of the solution of the case of a continuous beam of three unequal spans, the conditions of each of which are purposely made as dissimilar to those of the others as possible. The lengths $\mathrm{AB}, \mathrm{BD}$, and $\mathrm{DF}$, have been assumed in the proportions of 4,2 , and 1 ; $\mathrm{A} B$ has been supposed loaded with a weight of $4, \mathrm{BD}$ with a weight of 1 , distributed over the span uniformly, and $\mathrm{DF}$ has been supposed unloaded.

The Author's diagrams of the curves of bending moment (Plate 12A, p. 190, vol. xxxii., Minutes of Proceedings Inst. C.E.) may be referred to as showing the lengths of the lines $B x$, D $y$, \&c., under different conditions. These curves were constructed from the algebraical equations, but they are also true curves of equilibrium, with the springing points at different levels, for the insistent weights on the different spans. Curves corresponding to these for Fig. $\mathrm{H}$ are indicated by the dotted lines $\mathrm{A} \mathrm{C}^{\prime} x^{\prime} \mathrm{E}^{\prime} y^{\prime}$. These curves may be derived from A C B E D by making $\mathbf{B} x^{\prime}=\mathbf{B} x, . \mathrm{D} y^{\prime}=\mathrm{D} y$, and erecting on the sloping lines A $x^{\prime}, x^{\prime} y^{\prime}$, vertical ordinates equal to the ordinates of the curves A CBED measured from the horizontal line ABD. That is, for any point $t$, the line $r t$ being produced, to cut $\mathrm{A} x^{\prime}$ in $t^{\prime}$, the vertical height $t^{\prime} r^{\prime}$ is made equal to $t r$. The bending moment at any point is thus equally represented by the line $r s$ of the curve 
A C B E D, or by the line $r^{\prime} t$ of the curve A $\mathrm{C}^{\prime} x^{\prime} \mathrm{E}^{\prime} y^{\prime}$. The reason for constructing the curves of equilibrium on the horizontal lines A B, B D, \&c., instead of on the sloping lines A $x^{\prime}$, \&c., is, that the sums of the ordinates, or areas of the curve of moments, can then readily be found for any positions of the points $x, y$, \&c.; while if these areas had to be obtained from curves drawn on the sloping lines, new curves of equilibrium would be required for each different position of the points $x, y$, \&e.

The lengths $\mathrm{B} x, \mathrm{D} y, \& \mathrm{c}$, may be approximated to by assuming the beam to be severed at the ends of the several spans, beginning with the second. For the beam supposed continuous over the first two spans only, D $y=$ zero, and the length $\mathrm{B} x$ can be readily found by balancing the areas of the derived curve $\mathbf{A} m n \mathrm{D}_{2}$, as already explained. If the beam be then supposed continuous over the first three spans, $\mathrm{B} x$ and $\mathrm{D} y$ may be determined after one or two trials by balancing the areas, and it will generally be found that $B x$ will only differ slightly from the value already found for the continuous beam of two spans. Another span having been added, or the beam being supposed continuous over four spans, B $x$ will scarcely require alteration, while $\mathrm{D} y$ will only require to be altered slightly. This process may be continued for any number of spans, and for any manner of loading. An examination of the curves on the Plate 12A already referred to shows, that the effect of loading any span is scarcely felt beyond the third or fourth span from the one loaded. In Fig. $\mathrm{H}$, the dotted line $\mathrm{A} m n \mathbf{D}_{2}$ is the derived curve for the beam supposed continuous over the first two spans only, and $\mathrm{B} x_{2}$ is the corresponding bending moment at $B$, which in this case requires to be altered by the quantity $x x_{2}$ to suit the assumption of continuity over three spans instead of two.

The appropriate lines $\mathrm{A} x, x y, \& c .$, having been determined, the points of contrary flexure and of greatest bending moment can be ascertained by inspection of the diagram.

'The slope and ordinate of the elastic curve of the beam at any point may also be found as follows:

Calling $\lambda$ the vertical ordinate $r s$ between the curvo of equilibrium and the lines $\mathrm{A} x, x, y, \& \mathrm{c}$., measured by the same scale as the spans A B, B D, \&c.; the bending moment $M$ is equivalent to $H \lambda$, and the equation of equilibrium is

$$
\mathrm{E} \mathbf{I} \frac{\Delta^{2} y}{\Delta x^{2}}=\mathrm{H} \lambda
$$

$x$ being put for the abscissa $A, t$, and $y$ for the ordinato of the 
elastic curve of the beam, $+x$ measured from $\mathrm{A}$ towards $\mathrm{B}$, and $+y$ measured downwards.

Hence,

$$
\begin{aligned}
\mathbf{E} \cdot \frac{\Delta y}{\Delta x} & =\mathrm{H}\{\mathrm{\Sigma}(\lambda \cdot \Delta x)+\mathrm{C}\} \\
& =\mathrm{H} \cdot \Delta x\left(\Sigma(\lambda)+\frac{\mathrm{C}}{\Delta x}\right)
\end{aligned}
$$

But by the construction $\Sigma(\lambda)$ is represented by the ordinate $t u$ of the curve $A K_{0} B_{1}$, \&c., and $A A_{0}$ represents the arbitrary constant or $\frac{C}{\Delta x}$, in this case negative; $v u$ is therefore equal to $\mathrm{\Sigma}(\lambda)+\frac{\mathrm{C} \text {, }}{\Delta x}$ and if $v u$ be put equal to $v$

$$
\frac{\Delta y}{\Delta x}=\frac{\mathrm{H} \cdot \Delta x}{\mathrm{E} \cdot \mathrm{I}} \cdot v,
$$

an equation which gives the slope of the elastic curve of the beam at any point.

And for the ordinate of the elastic curve

$$
y=\frac{\mathrm{H}(\Delta x)^{2}}{\mathrm{E} \cdot \mathrm{I}} \mathbf{\mathrm { I }}(\nu)
$$

it being assumed that $y=0$ when $x=0$, or that the summation is commenced from the point $A$.

In reference to the above process it should be remarked that by making $t u$ equal, on the appropriate scale, to the sum of all the ordinates $r s$, from A up to and inclusive of $r s, t u$ thus really represents the sum of the heights of elementary parallelograms, or area $\mathrm{A} \mathrm{Cr} s$ divided by $\Delta x$, not up to $r s$, but up to a vertical line half-way between $r s$ and the next line of division further from $A$.

If the number of parts into which $\mathrm{AB}, \& \mathrm{c}$., is divided be considerable, the inaccuracy is slight: in order to be quite accurate, the ordinate $t u$ should be plotted half a division further from $A$ than the ordinate $r s$, and $t u$ will then represent the area of the curve AC $r s$ up to a point of the beam, corresponding to the point $u$ of the curve $\mathrm{A} \mathrm{K}_{0} \cdot \mathrm{B}_{1}$, \&c. A similar remark applies to the ordinates $v u$ of the curve $A K_{0} B_{1}$, the sum of which represents, on the appropriate scale, the ordinate of the elastic curve; this sum $u p$ to any point $t$ should be equal to the area contained between the straight line $A_{0} B_{0} D_{0}$, \&c., and the curve $A K_{0} B_{1}$ from $A$ up to the point $t$. A correction like that above described may be applied here, but as small errors are cumulative in integrating twice, it will be more accurate to measure by scale the ordinates of the curve $A K_{0} B_{1}$, \&c., and enter these measurements in the summation which expresses the ordinates of the elastic curve.

For any of the spans acted on only by a definite load at a given [1871-72. N.s.] 
point, the curve of equilibrium is two straight lines as explained (p. 86), the horizontal thrust being the same as for the other spans. If some of the spans are unloaded, as the span D F, Fig. H, the curve of equilibrium for one of these spans is a straight line D F joining the points of support; this line may be considered as representing a strut without weight, which conveys the horizontal thrust across the span, and vertical lines upwards or downwards, as may be necessary, will require to be drawn as before, in order to construct the derived curve and balance the areas.

If one or more of the points of support is not at the same level, and the continuous beam originally straight, the balancing of the areas will require to be arranged so that at the point corresponding: to the support which is out of level a small overplus may be left equal to the appropriate area which will represent the amount of difference of level. If $y$ in the above formula be put equal to this difference of level, $(\Delta x)^{2} \Sigma(\nu)$ will be the overplus of area.

To adapt the method to a continuous beam of varying section, it is only necessary in constructing the derived curve $A K_{0} B_{1}$, \&c., to substitute $\frac{r_{0} s_{0}}{I_{0}}, \frac{r_{1} s_{1}}{I_{1}}, \& c$., in the summations, instead of $r_{0} s_{0}, r_{1} s_{1}, \& \mathrm{c}$.; $I_{0} I_{1}, \& c$., being the values of $I$ for the cross section of the beam at the different points. $\mathrm{B} x, \mathrm{D} y, \& \mathrm{c}$, having been arranged so that the areas balance with these substitutions for $r_{0} s_{0}, r_{1} s_{1}$, the bending moments will be equal to the horizontal thrust multiplied by the ordinates $r_{0} s_{0}, r_{1} s_{1}, \& c$.

If the section of the beam.varies in such a manner that $I$ is everywhere proportioned to the strain, the values of $\frac{r_{0} s_{0}}{\mathrm{I}_{0}}, \frac{r_{1} s_{1}}{\mathrm{I}_{1}}, \& \mathrm{c}$., are constant, and the derived curve is a series of straight lines; the areas to be balanced are triangular, and if the proportion, or the constant $\frac{r s}{I}$, is the same for each piece of the beam lying between the points of contrary flexure, all the straight lines will cut the axis $A_{0} F_{0}$ at the same angle, and the approximation may be made with much greater facility than if the section of the beam were uniform.

On Fig. $\mathrm{H}$, the straight lines $\mathrm{A} T, \mathrm{~T} U, \mathrm{U} \mathrm{F}^{\prime}$, have been drawn so that the angles $\mathrm{T} e \mathrm{~B}_{0}, \mathrm{~T} g \mathrm{~B}_{0}$, and $\mathrm{U} k \mathrm{D}_{0}$ are equal to one another; and the areas $\mathrm{A} \mathrm{A}_{0} e, f \mathrm{~B}_{0} g$, and $h \mathrm{D}_{0} k$ are respectively equal to the areas $e \mathrm{~T} f \mathrm{~B}_{0}, g \mathrm{U} h \mathrm{D}_{0}$, and $k \mathrm{~F}^{\prime} \mathrm{F}_{0}$. The points of contrary flexure $\alpha^{\prime}$ and $\beta^{\prime}$, where the bending moment is zero, are 
determined by drawing the lines $\mathrm{T} \alpha^{\prime}, \mathrm{U} \beta^{\prime}$ through $\mathrm{T}$ and $\mathrm{U}$ parallel to $\mathrm{A}_{\mathbf{0}}$ : these lines will cut the curve of equilibrinm in the points $\rho^{\prime}$ and $\sigma^{\prime}$. Joining the points $\mathbf{A}$ and $\rho^{\prime}$, the line $\mathbf{A} \rho^{\prime}$ produced will cut the line $\mathrm{B} x$ in the point $x_{1}$, and the points $x_{1}$ and $\sigma^{\prime}$ being joined, the line $x_{1} \sigma^{\prime}$ produced will cut the line $\mathrm{D} y$ in the point $z$. B $x_{1}$ and $\mathrm{D} z$ are the bending moments at $\mathrm{B}$ and $\mathrm{D}$ on the above supposition. It appears that in the case represented by Fig. $\mathrm{H}$, which is probably an extreme one, the condition that the section varies as the strain, instead of being constant, involves an alteration of the points $x$ and $y$ to $x_{1}$ and $z$. On the whole, the bending moments of the large spans are not materially altered, and the Author observes that Mr. Heppel has made a similar remark. In the first case the stress per square inch on the outer surfaces of the beam would be of the same amount throughout; in the second it would vary as the bending moment.

Should it be desired to obtain the pressures on the piers, this may be done by taking moments for each pier successively, beginning with the end span, and including the moments $\mathrm{H} \times \mathrm{B} x, \mathbf{H} \times \mathbf{D} y, \& \mathrm{c}$., as follows :

Let $W_{1}$ be the load on the first span, and $p$ the horizontal distance from $\mathbf{B}$ of the vertical line passing through the centre of gravity of $W_{1}$. In like manner for the second span, let $W_{2}$ be the load, and $q$ the horizontal distance from $D$ of the vertical line which passes through the centre of gravity of $W_{2}$ : and let $W_{3}, W_{4}$, $\& c ., r, s, \& c$., be corresponding quantities for the succeeding spans. Then calling $s_{1}, s_{2}, s_{3}, \&$ c., the lengths of the spans reckoning from A towards the right, and $r_{0}, r_{1}, r_{2}, r_{3}$, \&c., the upward pressures at the piers beginning from $A, r_{0}$ being the upward pressure at $A$, if moments be taken round the ends of the 1st, 2nd, 3rd, \&c., spans successively, the following equations are obtained :

$$
\begin{gathered}
\mathrm{W}_{1} p+\mathrm{H} \cdot \mathrm{B} x-r_{0} s_{1}=0 \\
\mathrm{~W}_{2} q+\mathrm{W}_{1}\left(p+s_{2}\right)+\mathrm{H} \cdot \mathrm{D} y-r_{1} s_{2}-r_{0}\left(s_{1}+s_{2}\right)=0 . \\
\& \mathrm{c} . .+. \quad \& \mathrm{c} . \quad . \quad . \quad . \quad=\& \mathrm{c} .
\end{gathered}
$$

From the first of these equations $r_{0}$ may be found, and by inserting this value of $r_{0}$ in the second equation, $r_{1}$ may be found, and so on for $r_{2}, r_{3}, \& \mathrm{c}$.

In these equations, $\mathrm{B} x, \mathrm{D} y, \& \mathrm{c}$, , are to be reckoned as positive if $x, y, \&$ c., are below the line $\mathbf{A ~ B}$; the moment of the horizontal thrust then tends to turn the system in the same direction as the weights $\mathrm{W}_{1}, \mathrm{~W}_{2}, \& \mathrm{c}$. In Fig. $\mathrm{H}, \mathrm{B} x$ is negative, and $\mathbf{D} y$ positive.

If only one span be considered, the method above described affords a simple means of proving all the propositions relating to 
stress and deflection, for a beam with the ends free, or with one or both ends ' fixed ;' and with the load either acting at a given point or uniformly distributed over the span.

The action of oblique forces will now be considered.

\section{Oblique Forces.}

From what has been said as to the effect of transverse stress on the curvature of an arch rib, it will be seen that what determines the change of curvature is the amount of bending moment, and that it is of no consequence whether this has been produced by the action of vertical or oblique forces if the amount be the same. The different conditions already investigated will still subsist under oblique forces, due care being taken to obtain the correct value of M, which can no longer be taken to be represented by the line P N (Fig. F), as in the case of vertical forces.

\section{Curved Dock Gate.}

As the condition of dock gates under the pressure of water is in a great degree analogous to that of the voussoir arch, or arch hinged at the centre, the first and most simple illustration of oblique pressures will be that of the curved dock gates of the Victoria Docks, described at vol. xviii., p. 445, of the Minutes of Proceedings of the Institution of Civil Engineers.

Fig. 15 (Plate 3) shows a plan of one leaf of this dock gate, the surface exposed to the pressure of the water being circular. The other surface is also circular, but not concentric with the first, the gate being 3 feet wide at the middle, and only 2 feet at the ends. The neutral line is shown by the centres of the small circles midway between the two surfaces. The span of the lock is 80 feet.

$\mathrm{C} s_{1} s_{2} \ldots \mathrm{B}$, being the surface exposed to the pressure of the water, if all the pressures on it be resolved parallel to $C D$, the resultant may be represented by $B D$, and will pass through the middle point $d$ of $\mathrm{B} D$. Similarly, if the pressures are resolved parallel to $B D$, the resultant may be represented by the length $C D$, and will pass through the point $c$ in the middle of this length. Draw the lines $d b$ and $c e$ respectively parallel to $\mathrm{CD}$ and $\mathrm{D} \mathrm{B}$, and therefore in the directions of these resultants. These lines will intersect one another in some point $a$, and if $a b$ be laid off equal to $\mathrm{D} d=\frac{1}{2} \mathrm{D} \mathrm{B}$, and $b f$ be drawn parallel to $c e$ and equal to $\mathrm{C} c=\frac{1}{2} \mathrm{CD}$, then $a f$ to the same scale will be the amount, and will be in the direction of the resultant of all the pressures along $\mathrm{C} s_{1} s_{2} \ldots \mathrm{B}$, and will be normal to this surface. 
To construct the curve of equilibrium, which will pass through the heel post $\mathrm{A}$, and some point $x$ in the meeting post, divide the neutral line into a number of equal parts-in the figure there are twenty-and draw the normals $p_{1} s_{1}, p_{2} s_{2}$, \&c., through the middle points of these divisions, cutting the outer surface in $s_{1}, s_{2}$, \&c. Then, since the scale used is such that the pressure D B is represented by $a b$ or $\frac{1}{2} \mathrm{D} \mathrm{B}$, the pressures on $s_{1} s_{2}, s_{2} s_{3}$, \&c., which are all equal, will be represented by $\frac{1}{2}\left(s_{1} s_{2}\right)$, and the normals will be the directions of these forces. Draw $x h$ parallel to $\mathrm{C} \mathrm{D}$, cutting the line $f a$ produced in $a^{\prime}$, and join $A a^{\prime}$, which will be the direction of the force passing through $A$. And if $f^{\prime} a^{\prime}$ be made equal to $f a$, and $f^{\prime} g$ be drawn parallel to $x h$ to cut $a^{\prime} \mathrm{A}$ in $g, g a^{\prime}$ will be the amount of the force at $A$, and $f^{\prime} g$ the force at $x$.

Draw $g h$ parallel to $f a$, cutting $x h$ in $h$, and $g h$ will be equal to $f a$ or $f^{\prime} a^{\prime}$. Iraw $h q_{1}$ parallel to $p_{1} s_{1}$, and equal to $\frac{1}{2}$ of $s_{1} s_{2}$, and from the point $q_{1}$, so found, draw $q_{1} q_{2}$ parallel to $p_{2} s_{2}$, and also equal to $\frac{1}{2}$ of $s_{1} s_{2}$. Continue this construction of the force diagram for the remaining normals $p_{3}, s_{3}$, \&c., and if correct, the last point so found will coincide with $g$.

The curve of equilibrium is then constructed by drawing from the point $r_{1}$, where the first normal $p_{1} s_{1}$ cuts the line $x h, r_{1} r_{2}$ parallel to $a^{\prime} q_{1}$, to cut $p_{t} s_{2}$ in $r_{2}$. From the point $r_{2}$ so found draw $r_{2} r_{3}$ parallel to $a^{\prime} q_{2}$ to cut $p_{3} s_{3}$ in $r_{3}$, and continue this construction, which will terminate by the last line drawn passing through the point $\mathrm{A}$, and coinciding with $a^{\prime} \mathrm{A}$.

In this manner the curves of equilibrium on the figure have been drawn.

The central dotted line shows the curve of equilibrium which passes through the centre of the meeting post, supposing the gates to bear on each other at that point. It will be observed that, except at the ends of this line, there is a slight amount of transverse stress tending to diminish the curvature of the gate. This stress attains a maximum in the centre of the leaf, where the curve of equilibrium is about 4 inches distant from the neutral line. By the remarks on Eq. (6a), if the gate be considered as a box girder, and the curve touched the outer surface, the compressive stress would there be approximately double of that due to the uniform compression if the curve of equilibrium were central, and at the opposite side the stress would be zero. But as the curve is only about $\frac{2}{9}$ ths of the distance to the outer surface, the compressive stress there will be increased to $1 \frac{2}{9}$, and at the opposite side will be diminished to $\frac{7}{9}$ ths of the stress due to the uniform compression. 
'I'his result depends on the point of contact of the gates, and these stresses might be altered to $1 \frac{1}{9}$ th and $\frac{8}{9}$ ths respectively by dubling the timber and making the point of contact about 3 inches nearer the centre of the circle than the middle of the meeting post.

In order that there should be no transverse stress, the curve of equilibrium should be midway between the outer and inner surfaces; this could be easily arranged in constructing gates, by making the thickness so as to satisfy this condition.

'The other dotted lines show eurves of equilibrium passing through points 3 inches distant from the outer and inner surfaces, and are intended to show how the stresses would be altered by an obstruction getting between the gates, and forcing the curve to pass through these points. It will be observed that in that case there would be a gradual increase of transverse stress up to the meeting post.

The curves of equilibrium for a straight gate, or gate of any other form, may evidently be drawn in a similar manner.

\section{Elliptical Caisson.}

As an example of transverse stresses on a closed figure, the curve of equilibrium of the elliptical caissons used in the foundations of the Thames Embankment near Westminster Bridge will now be investigated.

These caissons (see "Builder" for 1864, p. 574) were elliptic cylinders, with a major axis of 12 feet 6 inches and a minor axis of 7 feet. They were made of wrought-iron plate about $\frac{3}{8}$ ths inch thick, and stiffened by angle irons.

Fig. 16 (Plate 3) represents a section of the caisson, $O$ being the centre and $\mathrm{A} \mathrm{O}, \mathrm{CO}$ the semi-axes.

If all the pressures on the quadrant $\mathrm{A} \mathrm{B} \mathrm{C}$ of the ellipse were resolved in a direction parallel to $A O$, the resultant would be a single force proportional to $\mathrm{CO}$, and passing through the middle of $\mathrm{CO}$. In like manner, the resultant of the pressures resolved parallel to $\mathrm{CO}$ would be a force proportional to $\mathrm{A} O$, and passing through the middle of $\mathrm{A} O$.

The intersection of the direction of these forces will therefore be the middle point $M$ of the line $\mathrm{A} C$.

Further, if $\mathbf{M} \mathbf{N}$ be drawn perpendicular to $\mathrm{A} O$, and the length M $\mathbf{N}$ made proportional to the length $A O$, and $N R$ drawn perpendicular to $\mathrm{C} \mathrm{O}$, and made similarly proportional to the length $\mathrm{C} \mathrm{O}$, then $\mathrm{MR}$ will be the resultant of all the pressures acting on 
the quadrant of the ellipse, to the same scale as $M N, N R$; and the triangles M N R, A O C, will be similar, and have equal angles, and $\mathbf{M ~} \mathrm{N}$ being perpendicular to $\mathrm{A} \mathrm{O}, \mathrm{M} \mathrm{R}$ will be perpendicular to $A C$. That is, the resultant $M R$ is at right angles to $A C$, and passes through its middle point $M$.

The pressures on the other quadrants of the ellipse may be considered as supplying forces at $\mathrm{A}$ and $\mathrm{C}$ to hold the quadrant A $\mathrm{B} \mathrm{C}$ in equilibrium. The force at $\mathrm{A}$ will be a force equal to $\mathrm{M} \mathrm{N}$, acting perpendicularly to $\mathrm{A} O$, and that at $\mathrm{C}$ a force $\mathrm{R} \mathrm{N}$, acting perpendicularly to $\mathrm{C} \mathrm{O}$. As the directions of these forces do not intersect one another in the line $M R$, but in a point $D$ outside it, they cannot of themselves balance the force M R.

But the cohesion of the metal supplies the force necessary to produce equilibrium, and the internal stress will be measured by the force so supplied.

Equilibrium would be produced by the addition at $\mathrm{A}$ of a twisting couple equal to $M N \times E D$, where $E$ is the point of intersec-

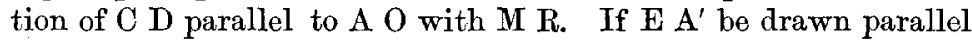
to $\mathrm{CO}$, cutting $\mathrm{A} O \mathrm{O}$ in $\mathrm{A}^{\prime}$, the addition of this twisting couple at A would transfer the force $M N$ parallel to itself to $A^{\prime}$, and there would be equilibrium, since then the directions of the forces $M N$, $\mathrm{R} \mathrm{N}$ would intersect one another in the line $\mathrm{M} R$.

In like manner, drawing $A \mathrm{E}^{\prime}$ parallel to $\mathrm{C} O$ to cut $M R$ in $\mathrm{E}^{\prime}$, if a twisting couple equal to $R \mathrm{~N} \times \mathrm{D} \mathrm{E}$, which is equal to the above couple $\mathrm{M} \mathrm{N} \times \mathbf{E D}$, were added at $\mathrm{C}$, the force $\mathrm{R} \mathrm{N}$ acting there would be transferred parallel to itself to $\mathrm{C}^{\prime}$, and the direction of the forces at $A$ and $C^{\prime}$ now intersecting one another in the line $M R$ produced, equilibrium would again subsist. And thus, taking any point $A^{\prime \prime}$ between $A$ and $A^{\prime}$, and drawing $A^{\prime \prime} E^{\prime \prime}$ parallel to $O \mathrm{C}$, and $\mathrm{E}^{\prime \prime} \mathrm{C}^{\prime \prime}$ parallel to $\mathrm{OA}$, if a couple represented by $\mathrm{M} \mathrm{N} \times$ A A $^{\prime \prime}$ were added at A to transfer the force there to $\mathrm{A}^{\prime \prime}$, and this couple being equal to $\mathrm{R} \mathrm{N} \times \mathrm{C}^{\prime} \mathrm{C}^{\prime \prime}$, a couple equal to $\mathrm{R} \mathrm{N} \times \mathrm{C}^{\prime \prime}$ would have to be added at $\mathrm{C}$ to transfer the force there to $\mathrm{C}^{\prime \prime}$ and. to produce equilibrium, the two added couples being together equal to the couple $\mathbf{M ~ N} \times \mathbf{E} \mathrm{D}$.

Any number of curves of equilibrium therefore can be drawn to pass through points in $\mathrm{A} \mathrm{A}^{\prime}$ and $\mathrm{C}^{\prime}$, each curve being subject to the condition that $\mathrm{A}^{\prime \prime}$ being one point, the other point $\mathbf{C}^{\prime \prime}$ is determined by drawing $\mathrm{A}^{\prime \prime} \mathbf{E}^{\prime \prime}$ parallel to $\mathrm{C} \mathrm{O}$ to cut $\mathbf{M} \mathrm{R}$ in $\mathbf{E}^{\prime \prime}$, and from $\mathbf{E}^{\prime \prime}$ drawing $\mathbf{E}^{\prime \prime} \mathrm{C}^{\prime \prime}$ parallel to $\mathrm{A} O$ to cut $\mathrm{C} \mathrm{C}$ in $\mathrm{C}^{\prime \prime}$.

Now, since the caisson is symmetrical, each quadrant has the same amount of pressure on it, and therefore the tangents to the 
curve of the caisson at $\mathrm{A}$ and $\mathrm{C}$ must be at right angles to one another both before and after strain; that is, the condition (3) must be satisfied, or

between the points $\mathrm{A}$ and $\mathrm{C}$.

$$
\mathbf{s}(\mathrm{M})=0
$$

This is also the only condition, as the caisson is at liberty to change its form, and cannot be considered as of invariable span.

To draw the curve of equilibrium, divide the quadrant CB A into any number of equal parts, ten in the figure, and at the centre of each part draw the normals $p_{1} q_{1}, p_{2} q_{2}$, \&c., which will be the directions of the pressures acting on the elementary arcs. From the point $R$ set off $R r_{1}$ parallel to the normal at $p_{1}$, and equal in length, to the same scale as $M N, N R$, to the pressure on one of the elementary arcs. From the point $r_{1}$, thus found, set off $r_{1} r_{2}=\mathbf{R} r_{1}$ and parallel to $p_{2} q_{2}$, and continue this construction, which will terminate at the point $M$.

Then if the points $r_{1}, r_{2}$, \&c., be joined with $\mathrm{N}, \mathrm{N} r_{1}, \mathrm{~N} r_{2}, \& \mathrm{c}$, will represent the amounts, and be parallel to the directions, of the curve of equilibrium at the different points. To draw the curve, from $\mathrm{C}^{\prime \prime}$ draw $\mathrm{C}^{\prime \prime} q_{1}$, parallel to $O \mathrm{~A}$, to cut $p_{1} q_{1}$ in $q_{1}$; from $q_{\mathrm{t}}$ draw $q_{1} q_{2}$, parallel to $\mathrm{N} r_{1}$, to cut $p_{2} q_{2}$ in $q_{2}$, and continue this construction, which will terminate by the last line drawn passing through the point $\mathrm{A}^{\prime \prime}$, and having the direction $\mathrm{A}^{\prime \prime} \mathrm{E}^{\prime \prime}$.

The point $C^{\prime \prime}$ was assumed tentatively, and the values of $M$ found from it. These were the lengths $\mathrm{N} r_{1}, \mathrm{~N} r_{2}, \mathrm{~N} r_{3}$, \&c., multiplied by tle perpendiculars on the directions of these forces at $q_{1}, q_{2}, q_{3}$, \&c., from the points $p_{1}, p_{2}, p_{3}$, \&c.

The position of $\mathrm{C}^{\prime \prime}$ was then altered until the line was obtained which satisfied the condition

$$
\mathbf{\Sigma}(\mathbf{M})=0 \text {. }
$$

In this instance, the pressure per square foot for a depth of 20 feet of water being 56 ton, for 1 foot in height of the caisson,

The force $M \mathrm{~N}$ is

and the force $\mathrm{N} \mathrm{R}$ is

The bending moment at $\mathrm{A}$ is 8.9 foot tons,

$$
\text { , }, \quad \text { at } \mathrm{C} \text { is } 6.6 \quad,
$$

Fig. 16 shows all the details of the construction, as explained above, for one of the quadrants.

The method of construction shows that when the eccentricity of the ellipse is small the curve of equilibrium is very nearly a circle, whose radius is the mean between the major and minor 
semi-axes of the ellipse. It follows from this that, if the shape of a boiler is not truly cylindrical, there may be considerable transverse, in addition to the tangential, stress; and if the deviation from the exact circle were greatest at the riveted joints, the stress would be greatest at the weakest parts. It has already been shown (p. 77), that when the curve of equilibrium touches the surface of an arch of rectangular section, the stress on the metal at the surface is quadrupled. The shell of a cylindrical boiler is an arch of this section, in tension instead of in compression, and therefore at an ordinary lap joint, or at any part where the deviation of form from the true circle amounts to only half the thickness of the plating, provided the deviation extends some little distance in the direction of the length of the boiler, the stress at the surface of the metal is four times that due to the pressure of the steam. This important result, showing how greatly a boiler may be weakened by an incorrectness of form too slight, especially if the boiler be made of steel, to be detected by the eye, is not generally known, so far as the Author is aware. There can be little doubt that incorrectness of form, the evidence of which is destroyed when a boiler explodes, is one of the chief causes of many of the boiler explosions which occur, from time to time, throughout the country.

As the circle is the curve of equilibrium for uniform normal pressure, it might at first sight be thought that it would always be the curve of equilibrium ; but an inspection of the figure will show that this cannot be the case. To illustrate the nature of the equilibrium, two of the elementary arcs at $p_{2}, p_{3}$ have been supposed to move parallel to their original positions until the curve of equilibrium passes through their centres. The equilibrium is not disturbed by this movement, but it is obvious that the directions of the forces acting on the ares differ considerably from the normals to the curve of equilibrium.

\section{Rigid Roof acted on ly Wind.}

In order to show the capacity of the methud above explained to deal with complex cases of structure, the next example chosen is the roof of the St. Pancras station. The form differs from both the circle and the parabola; the section of the rib varies to some extent near the springing; and, as the roof will be considered to be strained not only by its own weight, but by the side pressure of the wind, the case is also one of oblique forces. The particulars from which Figs. 17 and 18 (Plate 3) have been prepared were taken from 
the description of the roof given by Mr. W. H. Barlow, in the Minutes of Proceedings of the Institution of Civil Engineers, vol. $\mathrm{xxx}$., p. 78 .

Each principal or rib consists of a lattice girder of equal top and bottom flanges, and the neutral line is therefore in the middle of the rib, and is represented on the figure by the centres of the small circles. This neutral line has been divided into forty equal parts. At the centre of each of these divisions a vertical line has been drawn to the level of the base line, and a weight has been supposed to act at each of these centres, equal to the weight of the roof for the length of that division, and for $\mathbf{1}$ foot longitudinally in the line of the station. That is, since the length of each of these divisions measured along the neutral line is 8.7 feet, the weight has been taken at 8.7 square feet of roof, added to 8.7 feet run of the weight of the rib. This weight is not the same for each of the divisions; the weight per square foot of the lower portion, which is hoarded and slated, having been taken, exclusive of the weight of the rib, at twice the weight of the upper or skylight portion.

For the two divisions at the springing, the weight is that of the rib alone, which is heavier below the part where the lattice girder terminates.

Underneath the lattice girder the value of I for the rib has been taken at double of that for the lattice girder. The base line or termination of the divisions has been placed at that point of the rib, near the bottom, beneath which no appreciable curvature or movement firom stress could possibly occur.

The following are the weights which have been assumed:

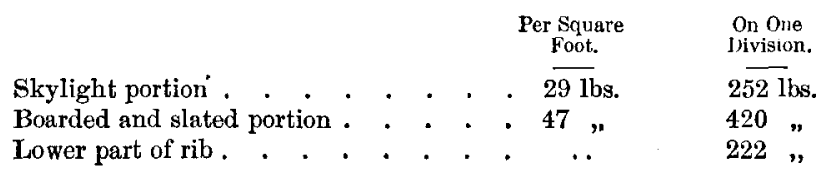

From these weights the lines on the lower diagram, Fig. 17, have been drawn. They represent the curves of equilibrium with the weight of the roof only. The line $(a)$, corresponding to the rib hinged at the crown, and the line $(b)$, corresponding to the rigid rib with the ends kept from spreading, were useful in fixing the position of the line (c), which is the curve of the rigid rib with the ends fixed. This latter line shows the actual stresses on the rib. It will be remarked that this line is contained everywhere within the depth of the rib, the neutral line of which it crosses and recrosses several times, the greatest deviation being incon- 
siderable. The neutral line of the arch rib therefore differs but slightly from the curve of equilibrium, and the transverse stresses arising from the weight of the roof itself are very small.

In Fig. 18, the horizontal force of the wind has been taken at $40 \mathrm{Ibs}$. per square foot, and has been treated in the following manner:-From the upper and lower edges of the back of each division horizontal lines have been drawn, the vertical distances between which, in feet, multiplied by $40 \mathrm{lbs}$., give the total horizontal force of the wind acting on the different divisions for 1 foot longitudinally of the roof. Each horizontal force has then been decomposed into an effective part, normal to the curve of the roof, and a non-effective part tangential to this curve. 'The effective part, passing normally through the centre of the division, has been decomposed into a horizontal and vertical component. The first has been considered as acting horizontally at the centre of the division, and the second as acting vertically there, and forming an addition to the weight of the division. ${ }^{1}$

The construction for this decomposition of forces is shown at the bottom of the Fig., to a scale of $100 \mathrm{lbs}$. to the inch. The total horizontal forces of the wind 07,08 , \&c., are laid off for the different divisions on the line $O X$. From the points 7, 8, \&c., perpendiculars are let fall on the corresponding normal lines which pass through $O$, the centre of the arch rib. The lengths of these perpendiculars represent the lost or non-effectivo forces, and the distances from the feet of the perpendiculars to $O$, the normal or effective forces. Perpendiculars let fall on the line $\mathrm{OX}$ from the ends of the normal forces determine the horizontal and vertical components of these forces.

The directions of the forces acting at each division were then found by laying off to the same scale vertical lines from the ends of the normal forces, each equal to the weight of the division of the roof to which it corresponded, as shown by the figures of reference. Lines joining the point $O$ with the upper ends of these verticals gave the directions of the forces at each point, and the full black lines which pass through the centre of each division are drawn parallel to these directions.

Having thus found the force acting on each division, and its

1 There is reason to believe that the Iaw of resistance here assumed, viz., that of the squares of the sines, errs in excess of the trutb. The construction is therefore a safe one, and the results would probably be approximately true for a considerably greater pressure of wind than that stated in the text. 
direction, the resultant of the whole of these forces was then ascertained as follows:

By taking moments with respect to the base line for each of the effective horizontal forces, their resultant or sum is equivalent to a force of $771 \mathrm{lbs}$. acting horizontally at a point 61.8 feet above the base line. In like manner, by taking moments with respect to $A$ of the vertical forces, their resultant is equal to a force acting vertically of $14,383 \mathrm{lbs}$. at a point distant 114.5 feet from $A$, or $5 \cdot 5$ feet from the centre towards A.

Combining these horizontal and vertical forces by construction, it was found that the resultant of the whole acted in the line $R R$, which cuts the neutral line of the arch rib in a point $C$ nearly midway between the centres of the divisions 19 and 20 .

In like manner, the resultant $P P$ of the forces acting between $C$ and $A$ was found, and the resultant $Q Q$ of those acting between $\mathrm{C}$ and $\mathrm{B}$.

As the point $\mathrm{C}$ is about $\frac{1}{10}$ th of the length of a division distant from the point midway between the centres of divisions 19 and 20, $\frac{1}{10}$ th of the weight of division 20 was added to the vertical force at division 19, and deducted from that at division 20 , in order to make the calculation of these forces correct.

The direction of the thrust at $\mathrm{C}$ was then determined by the method of drawing the curve of equilibrium for oblique forces. As a first approximation, the curve was assumed to pass through the centre of the arch rib at the crown. A point in the line $R \mathbf{R}$ was found such that when it was joined by straight lines with the points $A$ and $B$, which straight lines cut the directions of $P$ and $Q$ in the points $E$ and $F$, the line $E F$ passed through the centre of the rib at the crown, and gave the direction of the thrust there for the rib considered as hinged at the top. The lines $\mathbf{E ~ A ,}$ F B were then the direction of the thrusts at A and B. The length $\mathrm{E} p$ was then cut off on the line $\mathrm{E} P$ proportional to the force P. From $p$ the line $p k$ was drawn parallel to $\mathbf{E}$ F, cutting $\mathrm{A} \mathrm{E}$ in the point $k$, and the length $\mathrm{E} \mathrm{L}=p k$ was laid off on the line $\mathbf{E} \mathbf{F}$ from the point $\mathbf{E}$, thus fixing the point $\mathrm{L} .{ }^{1}$

The force diagram was then constructed by drawing to scale (3,000 lbs. to the inch), from the point $\mathrm{L}$, the line $\mathrm{L} a$ equal to the force acting on the division 19 , and parallel to its direction: from the end of this line the length $a b$ was drawn equal to the force at 18 , and parallel to its direction, and so on, the end of the last line coinciding with the point $k$ in the line A $\mathbf{E}$.

${ }^{1}$ A somewhat simpler construction is explained at page 113. See Note, p. 114. 
Lastly, the curve of equilibrium was constructed by drawing from the point $p_{\mathbf{1}}$, where the line of force at 19 cuts the line $\mathrm{E} \mathrm{F}$, the line $p_{1} p_{2}$, parallel to $\mathrm{E} a$, cutting the direction of the force at 18 in the point $p_{2}$, from $p_{2}$ the line $p_{2} p_{3}$, parallel to $\mathrm{E} b$, cutting the direction of the force at $\mathbf{1 7}$ in $p_{3}$, and so on, until the construction terminated by the last line drawn coinciding with the line $\mathrm{A} \mathrm{E}$, and therefore passing through the point $\mathrm{A}$.

Having thus drawn the curve of equilibrium for the arch hinged at the crown, as shown by the dotted line (a), it was found that this line almost exactly satisfied the condition

$$
\Sigma(M \cdot y)=0 ;
$$

the raising of the line at the crown, in order to exactly satisfy this condition, as shown by the upper dotted line $(b)$, being scarcely perceptible. Thus the curve for the rib supposed rigid, and the feet kept from spreading, was obtained.

The curve for the rigid arch with the ends fixed was then constructed after a few preliminary trials, and is shown by the dotted line $(c)$. The springing points of the curve requiring to be raised to $A^{\prime}$ and $B^{\prime}$, and the vertex to be lowered, to satisfy (3) and (6); the point of intersection of the thrusts at $\mathbf{A}$ and $B$ with the line $R R$ was altered slightly, and therefore also the position of the line $\mathrm{FF}$, the points $\mathrm{E}$ and $\mathrm{F}$ being changed to $\mathrm{E}^{\prime}$ and $F^{\prime}$. To avoid altering the force diagram, the line $\mathbf{L} \mathbf{E}^{\prime \prime}$ was drawn parallel to $\mathbf{E}^{\prime} \mathbf{F}^{\prime}$, and $k \mathrm{E}^{\prime \prime}$ parallel to $\mathrm{A}^{\prime} \mathrm{E}^{\prime}$, thus fixing the point $E^{\prime \prime}$, and the curve of equilibrium was then constructed by drawing the lines parallel to $\mathrm{E}^{\prime \prime} a, \mathrm{E}^{\prime \prime} b$, \&c., instead of $\mathrm{E} a$, E $b, \& c$. A similar construction was followed for the other point $\mathbf{F}$.

In drawing these curves, as the directions of the forces at 6,7 , 8, \&c., were not vertical, allowance for this had to be made in the values of M. Vertical lines were therefore drawn on the force diagram through the points $a, b, c, \& c$., to cut the line $\mathrm{EL}$, and any point, as $(m)$, was treated as if it formed part of a diagram, where the lines $a b, b c, c d$ were vertical, and the thrust therefore $\mathrm{E} m^{\prime}$, instead of $\mathrm{E} \mathrm{L}$; the lengths of the vertical lines $\alpha \beta, a \gamma$ corresponding to that point and representing $M$ were multiplied by the ratio $\frac{\mathrm{E} m^{\prime}}{\mathrm{EL}}$, before being put in the summation, and a similar corresponding ratio was used for the other points, where the forces were not vertical.

Referring to the line (c), it will be observed that a maximum stress occurs near the middle of the 11 th division, at which point 
the compressive force $\mathbf{F}$ is 1.36 tons per square inch, and the compressive stress on the upper part of the rib caused by the bending moment is 2.72 tons per square inch, making together a compressive force on the upper part of the rib of 4.08 tons per square inch.

The other maximum stress occurs at or near the centre of division 36, and is in like manner 1.88 ton per square inch for $F$, and $2 \cdot 26$ tons per square inch for the bending moment, giving together a compressive force at the under part of the arch rib of $4 \cdot 14$ tons per square inch.

The bending moments or transverse strains to be overcome in producing fixity of the rib at the springing are :

At the point $\mathrm{A}$ -

(Horizontal thrust $=42 \cdot 2$ tons) $\times 12 \cdot 4$ feet $=522$ foot tons; and at the point $\mathrm{B}$ -

(Horizontal thrust $=52$ tons) $\times 12 \cdot 4$ feet $=643$ foot tons.

The difference between these horizontal thrusts is the effective horizontal pressure of the wind on the space between the ribs.

\section{Rigid Arch Braced and acted on by Wind.}

When, as in the case of many roofs supported on walls or pillars, the ahutments are not constructed to withstand the thrust of the principals, and a horizontal tie-bar becomes necessary, this is generally raised above the level of the points of support, either for the sake of headway or for appearance, and its ends are connected with the apex and points of support by inclined tie-rods. The curve of equilibrium, including the action of these braces, can still be drawn, and will generally differ in form from the curve of continuous curvature already examined.

Taking the simple form of bracing shown by the full lines on Fig. 19, Plate $3 \mathrm{~A}$, and drawing the lines $\mathrm{D} \mathrm{F}, \mathrm{E} \mathrm{G}$, at right angles to the lines $\mathrm{A} \mathrm{C,} \mathrm{B} \mathrm{C,} \mathrm{through} \mathrm{their} \mathrm{middle} \mathrm{points} \mathrm{K}$ and.L, the curves of equilibrium for different positions of the ends $\mathrm{D}, \mathrm{E}$, of the tie-bar may be compared by assuming these points to be always at the same level, and to move along the lines D F, E G. If the points $D$ and $E$ are situated in the line $A B$, the curve of equilibrium passing through the points $A, C, B$ is the same as that found for fixed abutments, by the process already described. As the tie-bar is raised above the level of A B, it appears, by the construction explained a little further on, that the strain on it is increased in the ratio of $\mathbf{C ~} M$ to $C N$, and the strains on the ties A D, D C are also increased. The curve of equilibrium changes 
its form to that of a pointed or Gothic $\operatorname{arch} \mathrm{AFCGB}$, the tangents to which, at the apex $\mathrm{C}$, intersect one another in an angle. This useful and economical form of roof is deserving of consideration, and is intended to be used, for the roof of the new joint station at Bristol, by Mr. Francis Fox, M. Inst. C.E. The curve becomes flatter and the strains are increased as $D$ approaches K. The principals of a roof, if constructed to these curves, would be without transverse strain if loaded equally on each half of the span; but if an additional weight, or force of wind, be supposed to act on one side $\mathrm{A} \mathrm{C}$, and not on the other. the curve of equilibrium would change to some other curve A $F^{\prime \prime}$ $\mathrm{C} \mathrm{G}^{\prime} \mathbf{B}$. 'The point $\mathrm{F}^{\prime}$ would be further from $\mathrm{K}$ than $\mathrm{F}$, and the point $G^{\prime}$ nearer to $L$ than $G$. If the neutral lines of the curves of the principals were originally A F C G B, there would then be an amount of positive or negative transverse strain, measured by the pressure along the curve multiplied by the perpendicular on the tangent, as already explained.

'The curve has been assumed to be drawn so as to pass through $\mathrm{C}$, and the transverse strain is, therefore, zero at that point: this amounts to supposing the arch to be hinged at the crown. If it is made rigid there, the curve $\mathrm{A}^{\prime} \mathrm{C} \mathrm{G}^{\prime} \mathrm{B}$ must, satisfy the condition of invariability of span. In order to do this, the curve, drawn by the method described in the former part of this Paper, will pass a little below the point $\mathrm{C}$, and the strain there will tend to increase the curvature.

If the middle points of the principals be directly connected to the points $D$ and $\mathbf{E}$, the rib may have any other shape, as A $\mathrm{P}^{\prime \prime} \mathrm{C} \mathrm{Q}^{\prime \prime} \mathrm{B}$ (Fig. 20, Plate 3A), and the transverse stress will be zero at $A, P^{\prime \prime}, C, Q^{\prime \prime}, B$ if the rib be considered as hinged at these points.

The curve of equilibrium corresponding to the position of the points $\mathrm{D}$ and $\mathrm{E}$ in this figure being $\mathrm{A} \mathrm{P} \mathrm{CQ} B$, if the points $\mathrm{P}^{\prime \prime}$, $Q^{\prime \prime}$, be farther from $D$ than $P$ and $Q$, the connections $P^{\prime \prime} D, Q^{\prime \prime} E$, will be ties; but if these points are nearer to $\mathrm{D}$ and $\mathrm{E}$ than $\mathrm{P}$ and $\mathrm{Q}$, as at $\mathrm{P}^{\prime}, \mathrm{Q}^{\prime}$, the connections $\mathrm{P}^{\prime} \mathrm{D}, \mathrm{Q}^{\prime} \mathrm{E}$ will be struts. In any case, curves of equilibrium A $R P^{\prime \prime} S C^{\prime \prime} B, A R^{\prime} P^{\prime} S^{\prime} \mathrm{C}$ $Q^{\prime} B$ can easily be drawn, and are the forms which the neutral lines of ribs ought to have in order to be without transverse strain if loaded equally on each half span. These curves are concave to the straight lines A $\mathrm{P}^{\prime \prime}, \mathrm{P}^{\prime \prime} \mathrm{O}, \& \mathrm{c} ., \mathrm{A} \mathrm{P}^{\prime}, \mathrm{P}^{\prime}, \mathrm{C}$, \&c., and the amount of their separation from these lines depends on the weight and arrangement of the loading.

If an additional load or force of wind be supposed to act on one 
side $\mathrm{A} \mathrm{C}$, and not on the other, the curves of equilibrium have their curvatures increased on the side $A \mathrm{C}$, and diminished on the other side C B. Were the ribs or principals made to the curves of equilibrium A R P", \&c., there would thus be slight transverse stresses, which would be zero at the points $A, P^{\prime \prime}, C, \& c$. , and increase to a maximum at the middle of the distances $A P^{\prime \prime}, P^{\prime \prime} \mathrm{C}, \& \mathrm{c}$. These stresses would be of opposite signs on opposite sides of the crown C.

If the rib be supposed to be made rigid throughout, it appears to the Author that in the event of unequal loading, the rib will be in the state of a curved continuous beam of four spans; and the transverse stresses, as compared with those on the rib supposed jointed at $\mathrm{A}, \mathrm{P}^{\prime \prime}, \mathrm{C}, \& \mathrm{c}$., will, as for an ordinary continuous beam, be reduced to about one half; the stresses at $P^{\prime \prime}, C$, and $Q^{\prime \prime}$ having a contrary sign to those at the middle points of the distances $\mathrm{A} \mathrm{P}^{\prime \prime}$, $\mathrm{P}^{\prime \prime} \mathrm{C}$, \&c.

In addition to the transverse stresses brought on the ribs by unequal forces acting on the two sides of the span, the stresses on the other parts of the frame will be altered. The following construction shows the method of drawing the curve of equilibrium for the braced arch acted on by oblique forces, and of ascertaining the stresses on the other parts.

Referring first to Fig. 19, where the points D and E are unconnected with the principals; having drawn an approximate circular are for the curve of equilibrium passing through $A$ and $C$, divide this are into a convenient number of equal parts, and draw lines $p_{0} q_{0}, p_{1} q_{1}, \& c$., through the centre of each division in the directions of the forces acting on each of these divisions, as already explained for the roof of the St. Pancras Station.

The upward pressures at $A$ and $B$ may be found by taking moments round $B$ and $A$ of all the forces acting on the structure, including the force of the wind, supposed to act on A C, and the forces at $A$ and $B$ necessary to equilibrate this. If the span of the rib be invariable, or the ends be fixed in position, but not in direction, the forces necessary to balance the effect of the wind on $\mathrm{A} \mathrm{C}$, are-

(1.) A horizontal force at $\mathrm{B}$ acting in the direction $\mathrm{BA}$, and equal to the total effective horizontal force of the wind.

(2.) Upward vertical forces at $A$ and $B$, of such amounts as will equilibrate the effective vertical force of the wind.

(3.) A couple whose moment is $\mathrm{W} \cdot w$, if $\mathrm{W}$ be put for tho effective horizontal force of the wind, and $w$ for the vertical height of its centre of action above A. Calling $s$ the length of the span 
$A B$, this couple is equivalent to an upward vertical force $\mathrm{W} \frac{w}{\mathbf{s}}$ acting at $B$, and an equal vertical force acting downwards at $A$, since the horizontal force of the wind cannot alter the sum of the upward forces at $\mathrm{A}$ and $\mathrm{B}$. The moment $\mathrm{W} \cdot w$ of these forces must be added in taking moments round $A$ and $B$. If the rib is ouly fixed in position at one end, as at $A$, and the other end $B$ is on rollers, the force equal and opposite to $W$ will act at $A$ instead of at $B$. On this latter supposition, the stresses on the ties are increased.

The stress on the horizontal tie-rod D E is found by taking the moments round $\mathrm{C}$ of the forces to the left of $\mathrm{C}$, including the force of the wind. 'The accuracy of the results may be tested by taking moments to the right of $\mathrm{C}$, including that due to the external forces already mentioned.

In order to draw the diagram of forces; on the horizontal line $\mathrm{A} \mathrm{B}$, and with any convenient scale, make $\mathrm{A} a$ equal to the horizontal strain on the tie-bar, ascertained as above, and draw the line $a b$ parallel to $\mathrm{D} \mathrm{C}$, cutting $\mathrm{A} D$ in $b ; b a$ will then be the stress on $\mathrm{D} C$, and $\mathrm{A} b$ on the tie $\mathrm{AD}$. From the point $b$ draw $b \mathrm{~T}$ vertical, and equal to the upward pressure at $A$. A $T$ will be the resultant of the forces $A b, b T$, acting at $A$, and will be the direction of the tangent to the curve of equilibrium at the point $A$. The force diagram is then made by drawing $\mathbf{T} r_{0}$ equal to the force at $p_{0}$, and parallel to its direction, $r_{0} r_{1}$ equal and parallel to the force at $p$, and continuing this construction until the last point, $k$, is arrived at. Drawing $k f$ horizontal and $\mathbf{T} f$ rertical, $k f$ is equal to the total horizontal force of the wind, and $T f$ to the sum of the vertical forces acting on the rib between $A$ and $C$, including the vertical components of the force of the wind.

The curve of equilibrium is constructed by drawing $\mathrm{A} p_{\mathrm{o}}$ in the direction A T to cut $p_{\mathrm{o}} q_{\mathrm{o}}$ in the point $p_{v}$, from $p_{\mathrm{o}}$ drawing $p_{\mathrm{o}} p_{\mathrm{s}}$ parallel to $A r_{\circ}$ to cut $p_{1} q_{1}$, in the point $p_{1}$ and continuing this construction for the other points. The last point should be coincident with $\mathrm{C}$, and the tangent to the curve there should be parallel to $\mathrm{A} k$.

For the other side of the span, having made $\mathrm{B} a^{\prime}=\mathrm{A} a$, and drawn $a^{\prime} b^{\prime}$ parallel to the tie $\mathrm{EC}$, draw $b^{\prime} \mathrm{T}^{\prime}$ vertical, and equal to the upward pressure at $B$, and $T^{\prime} T^{\prime \prime}$ horizontal, and equal to the external force necessary at $B$ to maintain equilibrium. The forces on this side being all vertical, draw $\mathbf{T}^{\prime \prime} f^{\prime}$ vertical, and equal to the sum of the forces acting on the rib from $\mathrm{C}$ to $\mathrm{B}$. $\mathrm{T}^{\prime \prime} \mathrm{B}$ will be the direction of the tangent to the curve of equilibrium at $B$, and $f^{\prime} \mathrm{B}$ parallel to its direction at $\mathrm{C}$; and by dividing $\mathrm{T}^{\prime \prime} f^{\prime}$ into [1871-72. N.s.] 
portions representing the forces on each of the divisions of $\mathbf{C B}$, the curve of equilibrium can be drawn between $B$ and $C .1$

In Fig. 20 (Plate $3 \mathrm{~A}$ ), where the points $\mathbf{D}$ and $\mathbf{E}$ are connected to the middle points of the principals, the upward pressures at $A$ and $\mathrm{B}$, and the stress on the horizontal tie-bar $\mathrm{DE}$ being ascertained as before, the stress on $A D$ is found by taking moments round $\mathrm{P}^{\prime \prime}$ to the left of $\mathrm{P}^{\prime \prime}$; the moment of the force along A D balancing the difference between the moment of the upward pressure at A, and the moments of all the forces, including the force of the wind, acting on A $\mathrm{P}^{\prime \prime}$. Make A $b$ equal to the stress thus found, and A $a$ equal to the horizontal stress on the tie-bar D E. Joining $b, a, b a$ will represent the force to be balanced by the forces of $\mathrm{D} \mathrm{P}^{\prime \prime}$ and $\mathrm{DC}$. If $\beta a$ be drawn parallel to $\mathrm{DC}$, and $\beta b$ parallel to $\mathrm{DP}^{\prime \prime}, b \beta$ will be the stress on $\mathrm{DP}^{\prime \prime}$, and $a \beta$ the stress on $\mathrm{DC}$. From $b$ draw $b$ ' vertical, and equal to the upward pressure at $A$, and construct from the point $T$ the diagram of forces for the part $\mathrm{A} \mathrm{P}^{\prime \prime}$. From the last point $r_{5}$, so obtained, draw $r_{5} \mathrm{R}$ equal and parallel to $b \beta$, and continue the construction from the point $R$ for the part $P^{\prime \prime} \mathrm{C}$. The curve of equilibrium is then constructed from the force diagram as before; $\mathrm{A} T$ and $\mathrm{A} r_{\mathrm{s}}$ being parallel to the directions of the tangent to the curve $\mathrm{A} \mathrm{P}^{\prime \prime}$, at the points $\mathrm{A}$ and $\mathrm{P}^{\prime \prime}$, and $\mathrm{A} \mathrm{R}, \mathrm{A} k$, parallel to the directions of the tangents to the curve $\mathrm{P}^{\prime \prime} \mathrm{S} \mathrm{C}$, at the points $\mathrm{P}^{\prime \prime}$ and $\mathrm{C}$, through which points the curve of equilibrium passes.

For the other side $\mathrm{CB}$, the construction is made in the same manner, care being taken to include the effect of all the forces necessary to maintain equilibrium as already explained.

Figs. 19 and 20 are drawn to scale, and represent a roof of a rise equal to one-fourth of the span, with the ends fixed in position, but not in direction; or without expansion rollers under the end of the ribs. The diagrams of forces on each side of Fig. 19 are also drawn to scale, and show the amounts of the forces acting on one of the ribs, which are supposed to be 20 feet apart, the weight of the roof to he $10 \mathrm{lbs}$. per square foot, and a gross horizontal force of wind of $40 \mathrm{lbs}$. per square foot, acting on $\mathrm{AC}$. This force has been decomposed into effective horizontal and vertical forces, in the same manner as described for the roof of the St. Pancras Station, and the curves of equilibrium on the diagrams are assumed to pass through the point $C$, the apex of the roof.

: By supposing tho points $\mathbf{D}$ and $\mathrm{E}$ to bo in the line $\mathbf{A} \mathbf{B}$, this method of construction may be used for cases like the St. Pancras roof, and is more simple in some respectis than the method referred to in the commencement of this Paper. 
For Fig. 20, forces equivalent to those for Fig. 19 are supposed to act on the roof, and the diagrams of force are drawn both for the curve $A \mathrm{P}^{\prime \prime} \mathrm{C} \mathrm{Q}^{\prime \prime} \mathrm{B}$, and also for the curve $\mathrm{A} \mathrm{P}^{\prime} \mathrm{C} \mathrm{Q}^{\prime} \mathrm{B}$; those for the latter curve being indicated by a small o placed before the reference letters, as ${ }_{0} T$.

A comparison of these diagrams shows that the stresses are much smaller for the first form than for the second form of roof. The perimeter of the former is, however, a little longer than that of the latter, and although this will slightly increase the surface of the roof, the diminution of the stresses will, on the whole, considerably lessen the cost.

On Fig. 20, the full curved lines are the curves of equilibrium for the roof supposed to be acted on only by symmetrical vertical furces. The curves of equilibrium as altered by the action of the wind, differ so slightly from these as to be nearly undistinguishable on the small scale of the diagram.

\section{Efffect of a Smald Aliteration of Span.}

The span of the arch rib has hitherto been considered as invariable, that being the condition assumed in arriving at Equation (6). But there are several circumstances which induce real or virtual alterations of span; and although these alterations are small, as compared with the length of the span, yet the fact of their occurrence shows that the condition of absolute invariability of span cannot be held to represent the state of an arch rib under strain. The smallness of the alterations, however, allows of the use of the principle of the superposition of small changes; that is, that the total alteration, if small, due to any number of causes, is the algebraical sum of the alterations due to each cause separately.

(1.) By a change of temperature the length of the rib is altered, and its ends, if free, would be displaced, horizontally and vertically, through small, yet measurable spaces, which spaces would be proportional to the amount of change of teniperature. If the abutments could be supposed to move through these small spaces, so that their reactions, which existed before the change, could be applied to the ends of the rib with the same intensity and in the same direction as before, the equilibrium would not be disturbed. But as the abutments cannot adapt themselves to these changes, the feet of the arrh rib will spread or contract, so as to rest on the same points of the abutments as before, and the span will thus be virtually altered.

(2.) A direct lengthening of the span would be produced if the 
thrust of the arch forced the abutments farther apart than they were previously to this thrust coming upon them. The amount of yielding of the abutments it is impossible to predict, as it depends on the nature of their foundations as well as upon the bulk and quality of the masonry or brickwork, and there are as yet searcely any data recorded for existing structures. In a bowstring girder, the lengthening of the tie by stress, or alteration of temperature, corresponds to a yielding of the abutments of an arch.

(3.) The thrust of the arch will compress and shorten the length of the arch rib itself, and will, as appears by the above remark, produce a virtual lengthening of the span. The amount by which the span is lengthened from this cause may be determined by dividing the neutral line of the rib, on a drawing to a moderately large scale, into a number of equal parts, and laying off on each of these parts a length equal or proportional to the length by which that part of the rib is compressed by the thrust. These lengths depend on the amount of thrust and the sectional area of the rib, and will be equal to one another if this area is everywhere proportional to the thrust. The horizontal projections of the lengths may then be found, and their sum will be the horizontal displacement of the end of the rib, or the virtual change of span.

While arches were constructed of masonry or brickwork, it was only thought necessary to measure the amount of settlement of the crown, which might indicate, though it could not separately measure, a yielding of the abutments as well as a compression of the arch ring. Greater precision was immaterial, for, by the number of its joints and the plasticity of the mortar, an arch accommodates itself to its own internal changes and to a slight yielding of its points of support. With cast-iron arches more minute observations are requisite, while for large arches of riveted wrought iron, where the structure approaches in incompressibility to solid wrought iron, it becomes an important practical question to determine how much the stresses on an arch rib may be altered by an assumed small alteration of the span. The process already described, by which the curve of equilibrium can be found so as to satisfy the condition of invariability of span, gives at once the means of ascertaining this alteration of the stresses.

For, in approximating to that curve, values of $\mathbf{M}$ are obtained for one or more curves differing slightly from it in position. For any one of these curves, $\Sigma\left(\frac{M}{I} \cdot b c\right)$, instead of being equal to zero, is a definite quantity; and this quantity may be substituted 
in Eq. (4), which, as appears by the remarks on that equation, may be written thus:

$$
h=\frac{1}{a} \Sigma\left(\frac{M}{I} \cdot b c\right) \Delta s .
$$

The value of $h$, the horizontal displacement, will thus be obtained for a curve whose apex is higher or lower than that of the curve which satisfies the condition of invariability of span by a small quantity, say $d$.

If, then, $\sigma$ be the assumed horizontal displacement, a curve of equilibrium must be found, which, putting $\sigma$ for $h$ in the above equation, will satisfy the condition-

$$
\sigma=\frac{1}{\alpha} \mathbf{\Sigma}\left(\frac{\mathrm{M}}{\mathrm{I}} \cdot b c\right) \Delta s . . . .
$$

Calling $\delta$ the height of the apex of this last curve above that of the curve of invariable span, since $d$ and $\delta$ are both small, $\delta$ may be approximately found by the proportion-

$$
h: d:: \sigma: \delta
$$

and the apex of the curve being thus determined, the curve may be drawn, and the process verified by ascertaining whether the values of $M$ will satisfy Equation (13).

Having thus obtained the curve corresponding to the displacement $\sigma$, the stresses are the values of $M$ belonging to this curve, and may be compared with those of the curve of invariable span.

To give an idea of the change of stress caused by a given small alteration of span, the case of a parabolic, and approximately of a circular, rib of uniform section and small rise, loaded with a weight uniformly distributed horizontally, admits of easy solution.

Let the semi-span $=\mathrm{S}$ and rise $=\mathrm{D}, \sigma$ and $\delta$ meaning as above. Also let $\mathbf{I}_{0}$ be the horizontal thrust for the rib with the ends not displaced, and $H_{1}$ the horizontal thrust for the curve of equilibrium corresponding to the displacement $\sigma$, and rise $\mathrm{D}+\delta$. Taking the origin of co-ordinates at one end of the rib, for any abscissa $x$, let $y$ be the ordinate of the rib or curve of equilibrium for the thrust $\mathrm{H}_{0}$, and $y^{\prime}$ the ordinate of the curve of equilibrium for the thrust $\mathbf{H}_{1}$.

Then

$$
\begin{aligned}
y & =\frac{\mathrm{D}}{\mathrm{S}^{2}}\left(2 \mathrm{~S} x-x^{2}\right) \\
y^{\prime} & =\frac{\mathrm{D}+\delta}{\mathrm{S}^{\prime 2}}\left(2 \mathrm{~S} x-x^{2}\right)
\end{aligned}
$$

and

$$
\mathrm{M}=\mathrm{H}_{1}\left(y^{\prime}-y\right)=\mathrm{H}_{1} \frac{\delta}{\mathrm{S}^{2}}\left(2 \mathrm{~S} x-x^{2}\right) \text {. }
$$


Since the rise is small, $\Delta s$ may be taken equal to $d x$, and it is easily found by integration that

$$
\Sigma_{u}^{s} \mathbf{M} \cdot b c \Delta s=\int_{0}^{s} \mathrm{H}_{1}\left(y^{\prime}-y\right) y d x=\frac{8}{15} \mathrm{H}_{1} \delta \mathrm{S} \mathrm{D}
$$

Substituting this value in $\mathrm{Eq}$. (13),

$$
\sigma=\frac{8}{15} \frac{\mathrm{H}_{1} \delta \mathrm{S} \mathrm{D}}{\alpha \mathrm{I}} . . . .
$$

In this formula $\mathrm{H}_{1} \delta$ is the bending moment at the crown of the arch, which by Eq. (2) may be put $=\frac{t_{0}}{t_{0}} \mathrm{I}$, where $t_{0}$ is the stress per square inch caused by this bending moment. Also $f$ may be put for the uniform compression $\frac{\mathrm{H}_{0}}{\mathrm{~A}}$ per square inch caused by the thrust $H_{y \cdot}$ Multiplying the numerator of $\sigma$ in (14) by $\frac{\mathrm{H}_{0}}{\mathrm{~A}}$, and the denominator by its equivalent $f$, and substituting $\frac{t_{\mathrm{o}}}{k_{0}} \dot{\mathrm{I}}$ for $\mathrm{H}_{1} \delta$,

$$
\sigma=\frac{8}{15} \cdot \frac{t_{0}}{f} \cdot \frac{\mathrm{H}_{0} \mathrm{~S}}{\mathrm{~A} a} \cdot \frac{\mathrm{D}}{k_{0}} .
$$

But $\frac{\mathrm{H}_{11} S}{\mathrm{~A}} \frac{\mathrm{S}}{\alpha}$ expresses the contraction of a bar of a length $\mathrm{S}$ under the force $\frac{H_{0}}{A}$, or, in other words, the approximate horizontal displacement of the end of the arch rib by the force $\mathrm{H}_{0}$. Calling this $\epsilon$,

and

$$
\begin{aligned}
& \sigma=\frac{8}{15} \cdot \frac{t_{0}}{f} \cdot \frac{\mathrm{D}}{k_{0}} \boldsymbol{\epsilon} \\
& t_{u}=\frac{15}{8} \cdot \frac{k_{0}}{\mathrm{D}} \cdot \frac{\sigma}{\epsilon} f .
\end{aligned}
$$

For an arch rib of 200 feet span, and 20 feet rise, the depth of the rib being 4 feet, and the cross section $I$, or box-shaped,

$$
\begin{aligned}
& k_{0}=24 \text { inches } \\
& S=1200, \\
& D=240,
\end{aligned}
$$

And if $f$ be 4 tons per square inch, and $\alpha=10,000$ for wrought iron,

$$
\begin{gathered}
\epsilon=\frac{1200}{10000} \times 4=\frac{1}{2} \text { inch nearly }, \\
t_{0}=\frac{3}{2} \sigma .
\end{gathered}
$$


If $\sigma=\epsilon$, or the change of span be equal to the displacement of the end of the rib by the compression.

$$
t_{0}=\frac{3}{4} \text { ton per square inch. }
$$

Or; the shortening of the span by the compressive force $f$ will be $\frac{1}{2}$ an inch, and the additional stress at the outer surface of the rib at the crown, caused by the virtual lengthening of the span from compression, will be $\frac{3}{4}$ ton per square inch.

Again, making

$$
\begin{gathered}
\boldsymbol{t}_{0}=f \\
\sigma=2 \frac{2}{3} .
\end{gathered}
$$

Or a yielding of the abutments of $2 \frac{2}{3}$ inches each would double the stress on the outer surface of the arch at the crown, since the total stress there would be $f+t_{0}$.

The moment of any horizontal thrust being equal to the moment of the weights,

$$
\mathrm{H}_{0} \mathrm{D}=\mathrm{H}_{1}(\mathrm{D}+\delta)=\text { moment of weights. }
$$

Substituting the value of $\delta$ from this equation in Equation (14), it becomes, writing $\mathrm{A} q^{2}$ for I,

$$
\sigma=\frac{8}{15} \cdot \frac{\mathrm{D}^{2}}{q^{2}} \cdot \frac{\mathrm{H}_{0}-\mathrm{H}_{1}}{\mathrm{H}_{0}} \cdot \epsilon ;
$$

from which it appears that $\left(\mathrm{H}_{0}-\mathrm{H}_{1}\right)$, or the loss of horizontal thrust, is proportional to $\sigma$, the extension of the span.

$$
\text { And if } \begin{aligned}
\mathrm{H}_{1} & =0 \\
\sigma & =\frac{8}{15} \frac{D^{2}}{q^{2}} \epsilon .
\end{aligned}
$$

In the numerical example which has been chosen, $q$ may be taken $=24^{\prime}$, and

$$
\sigma=26 \frac{2}{3} \text { inches }
$$

would be the movement of each abutment when the arch had no horizontal thrust, but was in the condition of a girder. It is needless to say that the rib would break long before this extension of span was re:shed, wrought iron not being sufficiently extensible to admit of the theoretical result boing obtained.

The change in length of a bar of wrought iron, by a change of temperature of $15^{\circ}$, being the same as for a strain of 1 ton per square inch, and $f$ being 4 tons per square inch, if an arch rib of the above dimensions had its temperature reduced $4 \times 15^{\circ}=60^{\circ}$ below the temperature at which its parts were put together, an additional stress would be caused at the outer surface of the rib at the crown of $:$ ton per square inch. And if the abutments also yielded each $\frac{1}{2}$ inch under the thrust, the stress would be increased 
from $f=4$ tons to $4+3 \times \frac{3}{4}=6 \frac{1}{4}$ tons per square inch, by the compression of the arch ring, the reduction of temperature, and the yielding of the abutments. The change of temperature may either increase or diminish the stress, according as it is a fall-or a rise; but the compression of the arch ring, and the yielding of the abutments, always conspire together to increase the stress. To overcome this action, a margin should be allowed in the factor of safety, or the span of the arch during construction might be made slightly greater than the distance between the abutments; and before the weight of the roadway was allowed to come on the ribs the span could be shortened by wedging or otherwise.

\section{Elastic Curve and Deflection of the Crown.}

The elastic curve, or the curve which the neutral line will assume under the stresses to which the rib is subjected, may be found in the following manner :-

(1.) Supposing the temperature of the rib to change, the length of each of the divisions of the neutral line will be altered by a small quantity, which will be proportional to the length; and the form of the rib, as altered by change of temperature, may be considered to be a magnified representation of its original form. Further, by taking, on a drawing of the neutral line of the arch rib the length of a division to represent the alteration of its length, the drawing itself will represent the changes of the different points by the alteration of temperature. That is, if by applying an appropriate scale the alteration of span by change of temperature be measured by the span itself, the rise of the rib to the same scale will measure the alteration of rise, and the horizontal and vertical displacements of any point of the arch rib by change of temperature may be found by applying the scale to the drawing of the neutral line.

(2.) The compression of the arch rib by the force $\mathbf{F}$ will cause all its points to be displaced horizontally and vertically through small spaces, which may be determined by the following construction, which assumes that the sectional areas as well as the forces are not uniform throughout the rib:-The amount of the force $F$ at any point can be ascertained by scale from the force diagram used in drawing the curve of equilibrium; and from this, and the sectional area of the rib, the length by which each of the divisions of the neutral line is altered by compression may be found. Taking any assumed point as an origin, a diagram can then be constructed, by drawing through 
this point a line parallel to the direction of the neutral line at the centre of the first division, and equal in length to the amount by which the length of that division is compressed by the force $F$, from the point so found, drawing a line parallel to the direction of the neutral line at the centre of the 2nd division, and equal in length to the amount by which the 2nd division is compressed by the force $F$. This process is to be continued until the last division is arrived at. The horizontal and vertical displacements of a point in the neutral line of the rib, at the centre of any division, will then be the horizontal and vertical projections of a line on this diagram, joining the origin with the middle of the line corresponding to the compression of that division. By this means the horizontal displacement of the end of the rib by the compression may be ascertained.

(3.) The conditions existing at the ends of an arch rib being given, that is, whether the span is invariable, or altered by a small quantity, namely, the amount of yielding of the abutment, and the alterations produced by compression and change of temperature, and whether the ends are free or 'fixed,' the proper curve of equilibrium to satisfy these conditions can be drawn by the method above described, and the values of $\mathbf{M}$ ascertained. The displacements of all the points of the rib by the bending moments $M$ can then be determined by Equations (4) and (5), which may be thus written :

$$
\begin{aligned}
& h=\frac{1}{a} \Sigma\left(\frac{\mathrm{M}}{\mathrm{I}} \cdot b c\right) \Delta s \\
& v=\frac{1}{a} \Sigma\left(\frac{\mathrm{M}}{\mathrm{I}} \cdot x\right) \Delta s ;
\end{aligned}
$$

or, in the case of vertical forces (Fig. F):

$$
\begin{aligned}
& h=\frac{\mathrm{H}}{a} \mathrm{\Sigma}\left(\frac{\mathrm{P} \mathrm{N} \cdot \mathrm{PQ}}{\mathrm{I}}\right) \Delta s \ldots . . \\
& v=\frac{\mathrm{H}}{\alpha} \mathrm{\Sigma}\left(\frac{\mathrm{P} \mathrm{N} \cdot \mathrm{A} \mathrm{Q}}{\mathrm{I}}\right) \Delta s . . .
\end{aligned}
$$

In reference to the limits between which the summations indicated by $\Sigma$ in these formulæ are to be taken, it is to be remarked that, referring to formula (15), if the summation be performed from A up to B (Fig. F, p. 79), and the result be substituted in this furmula, the value of $h$ is the total horizontal spread of the feet of the arch rib, and this spread must be equal to the sum of the displacements of $A$ and $B$, from the yielding of the abutments and the alterations produced by compression of the rib and change 
of temperature. Likewise, if the vertex $\mathrm{C}$ were considered as a fixed point, the displacement of the point $A$ would be the value of $h$ as obtained by performing the summation between $\mathrm{A}$ and $\mathrm{C}$, and substituting this in formula (15); and, in like manner, the displacement of $B$ would be obtained by means of the summation from $\mathrm{C}$ to $\mathrm{B}$. But the actual displacements at $\mathrm{A}$ and $\mathrm{B}$ from the causes enumerated above may, and probably will, in most cases differ from the displacements obtained by considering the vertex $\mathrm{C}$ as a fixed point. It will not, however, be difficult to find, by a trial or two, such a point, say $\mathrm{C}^{\prime}$, that the summation from $\Lambda$ to $\mathrm{C}^{\prime}$ being substituted in formula (15), the resulting value of $h$ will be the actual displacement of $A$. This point $C^{\prime}$ having been found, since the total spread is equal to the sum of the displacements at $\mathrm{A}$ and $\mathrm{B}$, the summation from $\mathrm{C}^{\prime}$ to $\mathrm{B}$ substituted in formula (15) will give for the value of $h$ the actual displacement of $\mathrm{B}$ from the above causes.

The elastic curve being then referred to the point (" as an origin, the displacement of any other point as $p^{I I}$ may be found by entering the difference between the summation from $\mathrm{A}$ to $\mathrm{C}^{\prime}$, and from $p^{\text {II }}$ to $\mathrm{C}^{\prime}$, in formula (15). The displacement of $p^{\mathrm{II}}$ may otherwise be found, as in the first method mentioned in p. 71, by performing the double summation indicated by $\Sigma \Sigma \mathrm{M} \Delta y \Delta s$, from $\mathrm{C}^{\prime}$ to $p^{\mathrm{II}}$, and substituting this in formula (15), instead of the single summation.

Similar remarks to the above apply to formula (16) for vertical displacements.

Having thus found the displacements of the different points, the elastic curve can be drawn through the new positions of the points so displaced, and the deflection of the crown will be simply the alteration in the rise of the arch rib.

In the case of the parabolic or circular rib of small rise, a formula for $v$ may be arrived at similar to that for $\sigma$ in Eq. (14).

Putting $d x=\Delta s$, it is found by integration that

$$
\Sigma_{0}^{s}(P N \cdot A Q) \Delta s=\int_{0}^{3 s} \mathrm{H},\left(y^{\prime}-y\right) x d x=\frac{5}{12} \mathrm{H}_{،} \delta \mathrm{s}^{2},
$$

and substituting this value in Eq. (16),

$$
v=\frac{5}{12} \cdot \frac{\mathrm{H}, \delta \mathrm{S}^{2}}{a \mathrm{I}}
$$

Combining this with (14),

$$
v=\frac{75}{96} \frac{\mathrm{S}}{\mathrm{D}} \sigma
$$


showing that the deflection is in proportion to the extension of span.

For the arch rib of the roof of St. Pancras Station, not acted on by wind, the virtual lengthening of the span by the compression of the arch rib, found by the construction described above, is $\cdot 23$ inch. Substituting this for $h$ in Eq. (15), and taking $\alpha=8000$ for riveted iron,

$$
I=46 \text { sq. inches } \times 36^{2}=59600, \Delta s=8 \cdot 7 \text { feet, or } 104 \text { inches, }
$$

and the horizontal thrust of the rib 46 tons; the resulting value of $\mathbf{\Sigma}(\mathbf{P N} \cdot \mathrm{PQ})$, the unit being one foot, is 166 . But it appears by Fig. 17, that an alteration of the vertex of the curve of equilibrium (c) by 1 foot, makes an alteration in $\mathrm{\Sigma}(\mathrm{P} \mathrm{N} \cdot \mathrm{PQ})$ of 900 , and for an alteration of 166 , the vertex of the curve would require to be shifted 1 foot $\times \frac{16 \frac{6}{9}}{9} \frac{6}{0}$, or $\cdot 18$ foot. This is not more than the thickness of the line on the figure, so that the values of $M$ as altered by the compression of the arch rib may still be taken from the curve (c). Making, then, for this curve, the summation $\mathbf{\Sigma}(\mathrm{PN} \cdot \mathrm{AQ})$ for the half rib, it is found that the + and - values, 423 and 446 , are so nearly equal that the sum may be taken as zero.

The deflection of the crown, therefore, will be unaltered by the transverse stresses brought upon the rib by its own weight.

And thus the deflection will be simply the diminution in the rise of the rib, caused by its own compressibility. This, taking $a=8000$ for riveted iron, is found, by a diagram constructed as already described, to be $\cdot 2$ inch.

As stated in Mr. Barlow's Paper, the observations made upon the rib itself gave from $\frac{3}{16}$ th to $\frac{1}{4}$ th inch for the deflections, so that the agreement with calculation is very close indeed.

Had the rib not been fixed at the ends, the value of $\Sigma(P N \cdot A Q)$, estimated from the curve $(b)$, would have been -1648 , the unit being one foot; and the deflection, calculated by substituting this value in Eq. (16), would have been $2 \cdot 4$ inches, in addition to the diminution of rise cansed by the compression of the arch rib.

\section{Direct Measurenevt of Stress.}

It would be somewhat difficult, by ordinary methods, to test satisfactorily the stresses on an arch rib, so as to compare them with calculation. Observations on deflection would give some information, but a model would necessarily be on a comparatively small scale, and the rib would require to be curved, yet as much as possible withont initial stress. 
The Author thinks that when it is desirable to know the stress which any part of a structure or model is sustaining, it is possible to ascertain this by direct observation.

The principle which underlies all investigations of stress, and which has been established by experiment, is that, for working stresses, extension or compression is proportional to stress; or, in other words, the nature of the material, and the extension or compression of a given length of it being known, the amount of stress can be at once determined.

In wrought iron, the extension on a length of 50 inches, with a strain of $\frac{1}{5}$ th ton per square inch, would be $\frac{1}{1000}$ th of an inch. This length is quite within the reach of exact measurement by means of magnifying glasses ; since, with a power of 50 , it would be equivalent to measuring $\frac{1}{2}$ th of an inch by the naked eye. If two microscopes, magnifying even less for iron, and considerably less than this for timber, were attached to a bar of the same material as the structure to be tested, so as to eliminate the effect of alterations of temperature, about 50 inches long, and were each capable of being moved a short distance along the bar by a micrometer screw, it is believed that stresses of $\frac{1}{5}$ th ton per square inch could be read off with certainty. For this purpose, it would only be necessary to furnish the eye-pieces of the microscopes with finelydivided scales. By means of the micrometer screw, having set the zeros of the scales nearly to coincidence with two marks made on the iron, at the part where the stress was expected to be the greatest, the readings of the scales before and during the testing would give the stresses in the most direct manner.

The exact value of the scales, in terms of the stress, could be determined, if necessary, by a preliminary experiment on the direct extension of a piece of the material.

By this means, the stress could be measured at that part of the structure where it was most important to measure it.

It might be said that, owing to the want of homogeneity of the material, the short piece, on which the observation was taken, would differ in extensibility from the part of the structure it was desirable to test. Put this objection is not of much force, since experiments on pieces of moderate length, show that the rate of extension for working stresses does not vary, in different specimens of the same material, to nearly the same extent as the breaking weight, which is probably determined by the imperfection of a portion of the material of very limited dimensions.

The measurement of the small extensions or compressions here proposed might even be useful in another way. 
It is found that the modulus of elasticity, and the breaking weight, are each greater for wrought than for cast iron, and greater for steel than for wrought iron; and it may therefore be said, though in a very general way, that the less the extensibility, the greater the breaking weight.

This view is borne out by Fig. 21, Plate $3 \mathrm{~A}$, which shows some results obtained from experiments when examined under this aspect.

Referring first to the experiments on tensile strain, which are distinguished on the figure by small dots, those for steel and wrought iron have been taken from the "Experiments on Steel made by a Committee of Civil Engineers" (1868-70), and those for cast iron from the Report "On the Application of Iron to Railway Structures" (1849). 'I'he fair line A A has been drawn to represent the average of all these experiments.

It will be remarked that wrought iron has about one half the extensibility, and fully three times the strength of cast iron; and that although the strength of steel varied considerably in the different specimens tried, the extensibility was very nearly the same for all-meaning by extensibility, the alteration of the unit of length by a force of one ton per square inch.

The remaining particulars on Fig. 21 have been taken from the experiments on cast-iron bars broken by transverse strain, which are recorded in the second of the above-mentioned, Reports, and the fair line B B has been drawn to represent average results.

For each experiment, the extensibility was taken as the reciprocal of the modulus of elasticity, in the ordinary formula which connects the central deflection of a bar with the weight of the load acting on it. The breaking stress per square inch was considered to be that on the outside surface in the middle of the bar, as deduced from the well-known formula, which counects the load, taken as the breaking weight, on the middle of the bar, with the tension on the outer surface in the middle of the span.

It has long been known that the breaking stress, deduced in this manner, was very much higher than any result that could be obtained from experiments on direct tensile strain; and this fact, whatever may be its cause, accounts for the want of coincidence between the lines B B and A A. Mr. W. H. Barlow has examined this subject minutely, ${ }^{1}$ and has arrived at the result, that in cast iron the breaking stress, deduced from transverse fracture, is about two and a quarter times that obtained from fracture by direct tension.

1 Vide Philosophical Transactions, vol. cxlv., p. 225, and rol. clxvii., p. 463. 
If the vertical ordinates of the line $\mathrm{B} B$ be reduced in this ratio, the line $\mathrm{C} C$ is the result; and this line, as will be observed, is nearly coincident with the line A A.

The subject of the connection between the breaking weight and the extensibility is deserving of further examination, as the experiments on wrought iron are few, and have probably been made on good specimens, whereas it would be desirable to examine experiments made on fair commercial samples; and should it be found, that as an inferior kind of wrought iron approaches to cast iron in its physical structure and breaking weight, it also approaches it in the scale of its extensibility for working stresses, an observation of the kind above mentioned, taken where the strain could be accurately determined by calculation, would give the means of ascertaining the quality of the iron used in a structure.

The communication is accompanied by a series of diagrams, from which Plates 3 and $3 \mathrm{~A}$ have been compiled.

[M. J. GaUdari, 

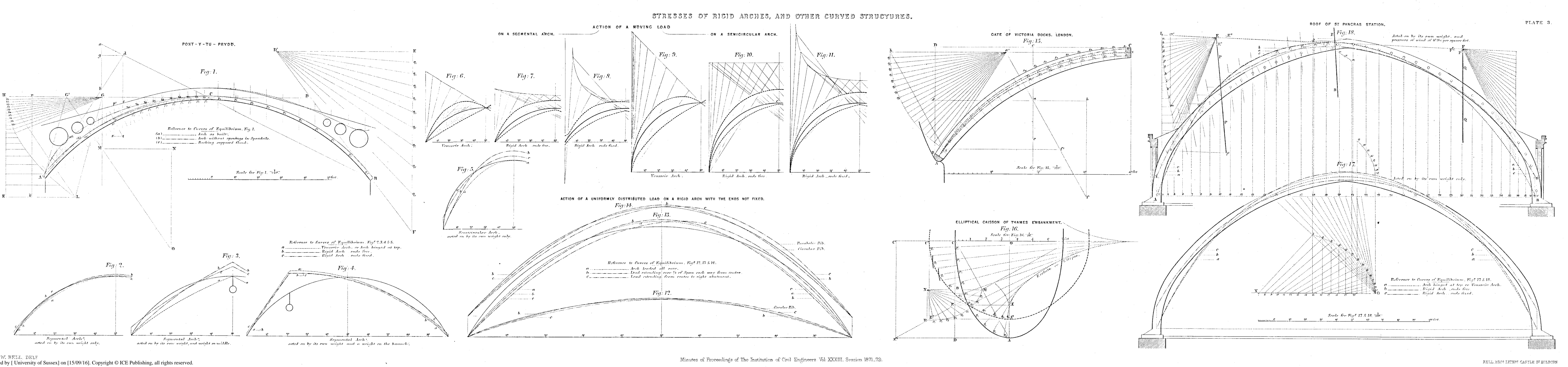
CONTINUOUS BEAM.

$F i g: H$

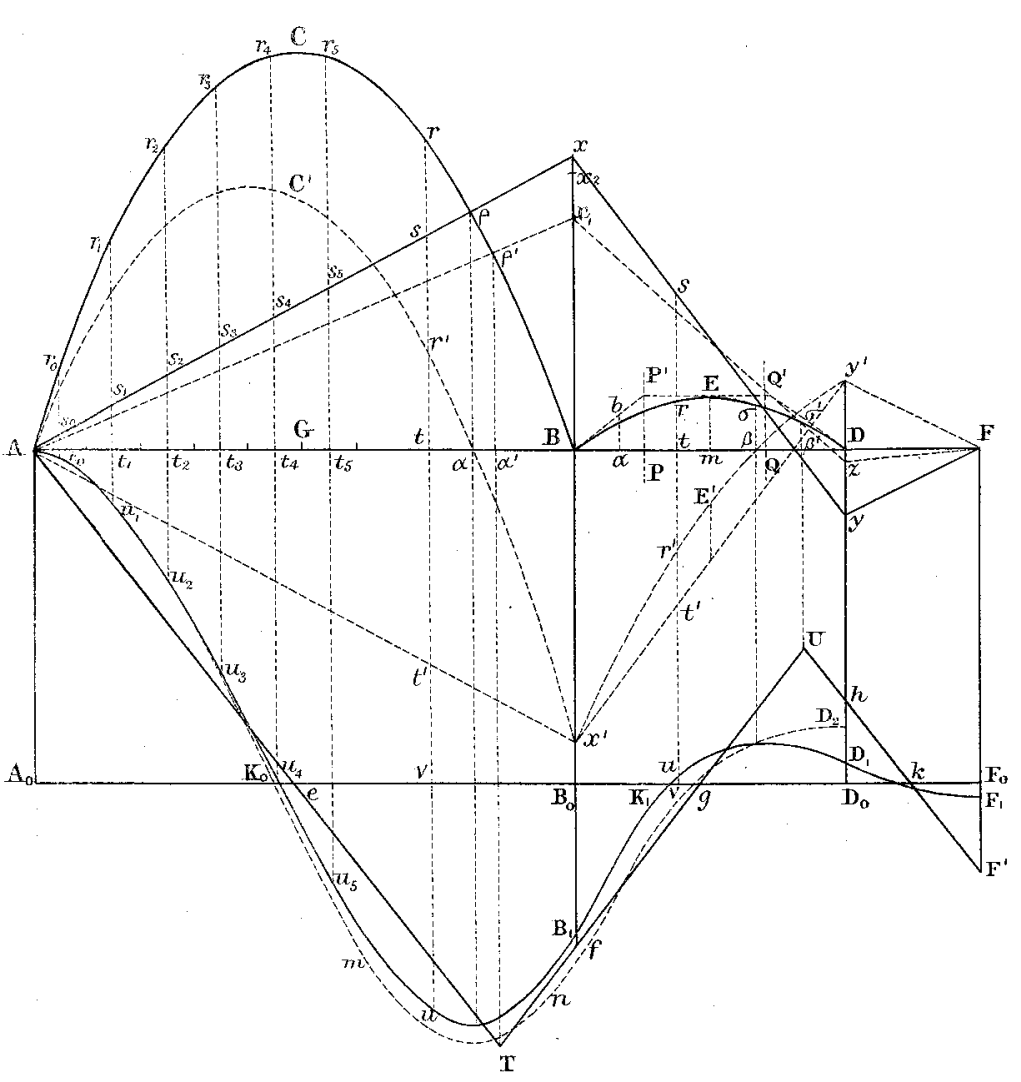

RIGID ARGH BRACED.

Fig: 19.

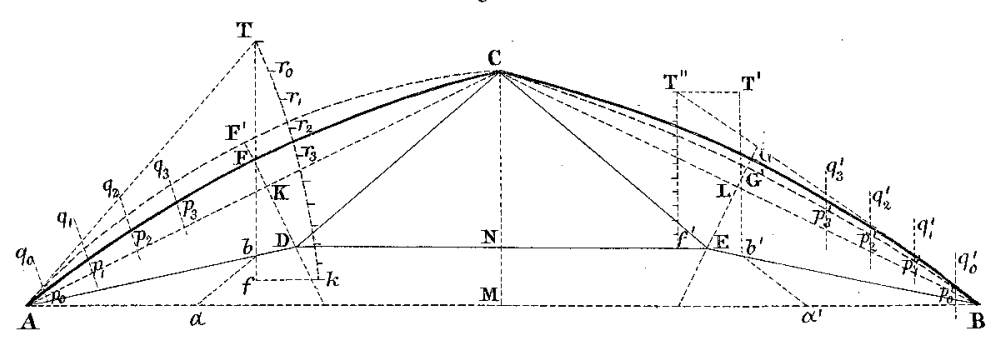

Fig: 20
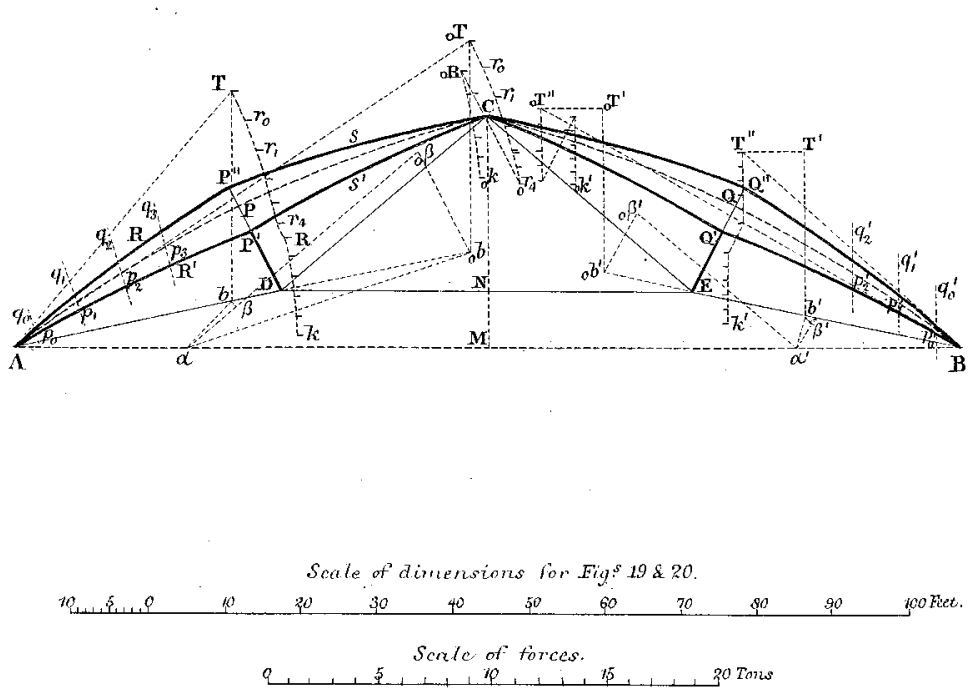

Fig: 21.

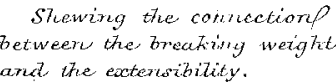

EXPLANATION OF MARKS: Fxperimerts ore tensize straiz marked' by dots.

Experiments or crast irone subjected to transwerse stratio

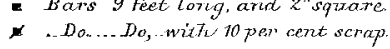

- .Do......Do. with 15 perer cent scrapo

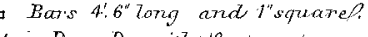

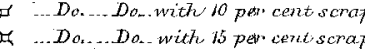

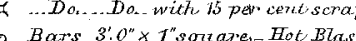

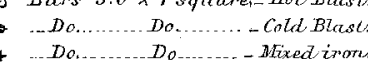
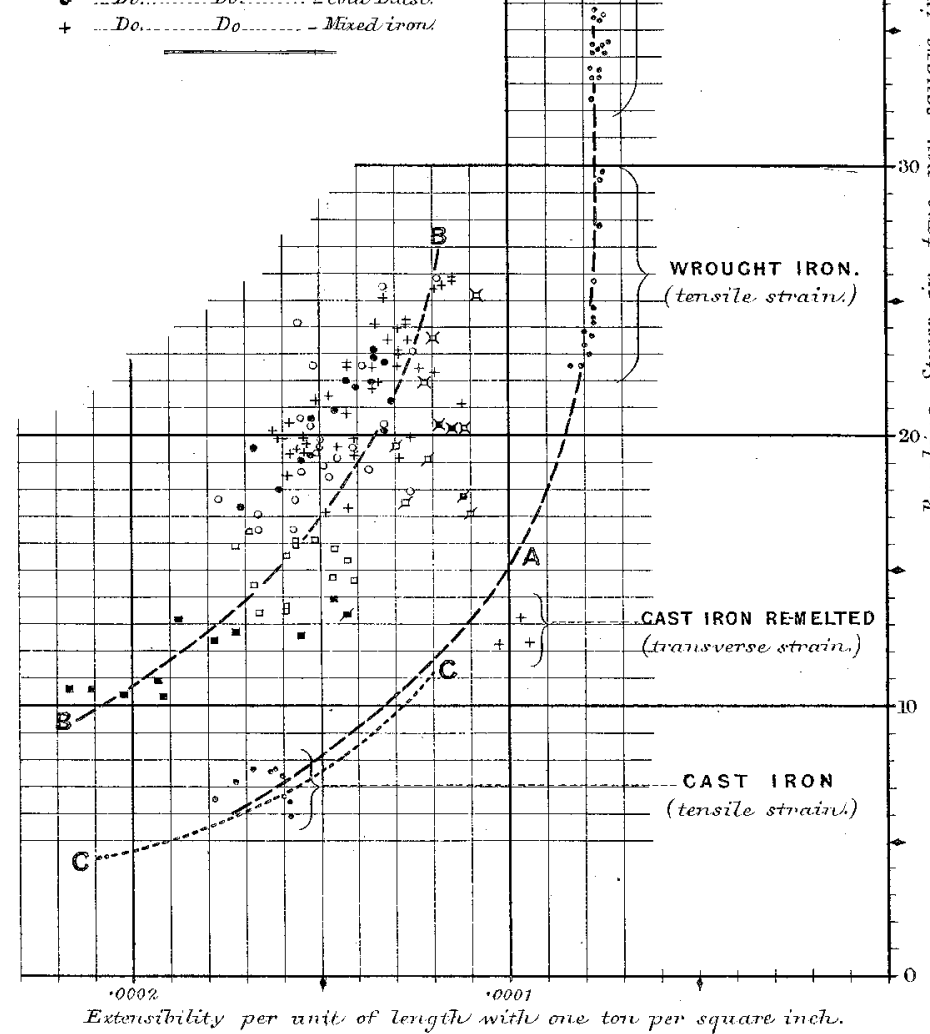

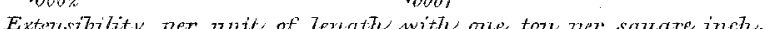

KREL, BROS. ITEATSE CASTIE ST HOTBORN 